\title{
2
}

\section{Color Psychology and its Effect on Hospital Design and Patient Treatment}

\author{
Arezou Eshaghabadi ${ }^{1}$, Pir Hossein Koulivand ${ }^{1,2^{*}}$, Hadi Kazemi ${ }^{1,3}$ \\ ${ }^{1}$ Shefa Neuroscience Research Center, Khatam Alanbia Hospital, Tehran, Iran \\ ${ }^{2}$ Department of Organizational Behavior Management, School of Management, Payam Noor University, Tehran, Iran \\ ${ }^{3}$ Pediatric Department, Medical Faculty, Shahed University, Tehran, Iran
}

\begin{tabular}{lcl}
\hline & Article Info: & \\
\hline Received: 18 Jun 2016 & Revised: 1 Oct 2016 & Accepted: 5 Nov 2016
\end{tabular}

\section{ABSTRACT}

Introduction: Environment color design includes all surfaces and interior furniture from the light and color to the feature and internal atmosphere, aesthetics and efficiency. Color design can play a role in the treatment procedure and efficiency. Considering that there is not a general and simple basis for environment color design, this essay states some principles to help complete color design in order to address most queries. If environment color design is produced carefully and through plans, it can develop the sense of health in medical facilities where patients are hospitalized for long terms. To get reasonable conditions in hospitals, we need to benefit from all current knowledge. Conclusion: Color design has a direct effect on people's spirits. Bright, subtle, and soft colors should be used and somber and saturated colors should be avoided. It seems necessary to use the combination of color with appropriate lights for all hospital's clients to orient themselves and find directions. This is especially important in the elderly patients who are a major part of hospital population and are hospitalized for long-term periods. The disabled and those with mental problems feel extremely in danger when surrounded within a closed space with strong colors, like orange and red. For children and teenagers, good lighting and color design can make a less organization-like and more pleasant and interesting place. For hospital staffs, proper color design and lighting can create a suitable environment to work effectively with delighted Spirit.

\section{Key words:}

1. Color

2. Color Therapy

3. Hospitals

*Corresponding Author: Pir Hossein Koulivand

E-mail:peirhossein@yahoo.com 


\title{
روانشناسى رنتَ و اثر آن بر طراحى بيمارستان و درمان بيمار
}

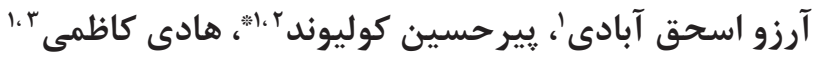 \\ 'مركز تحقيقات علوم اعصاب شفا، بيمارستان خاتم الانبياء، تهران ايران \\ rكروه مديريت رفتار سازمانى، دانشكده مديريت، دانشَاه يِيام نور، تهران، ايران \\ 'بخش اطفال، دانشكده يزشكى، دانشعاه شاهد، تهران، ايران
}

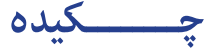

مقدمه: طراحى رنگ محيط، شامل تمام سطوح و وسايل داخلى از نور و رنت تا نما و فضاى داخل،

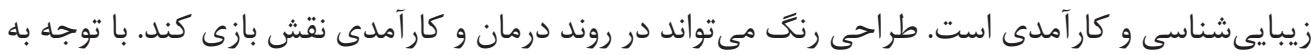

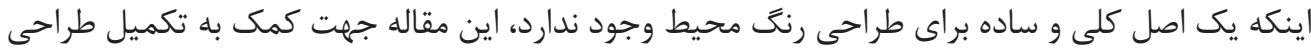

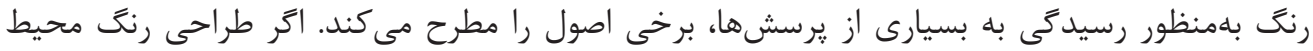

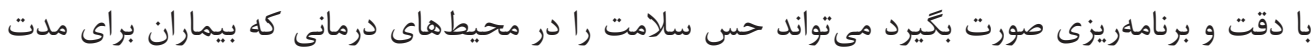

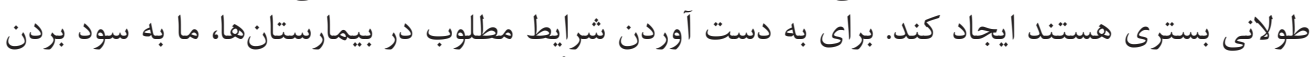

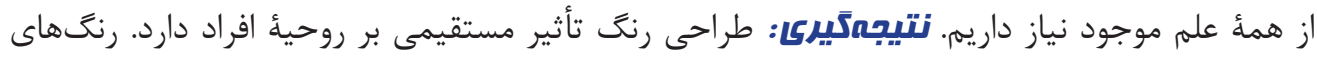

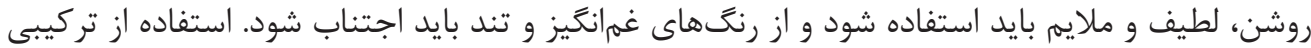

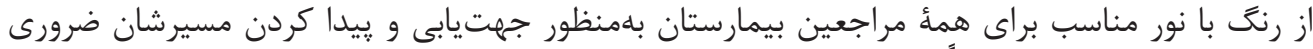

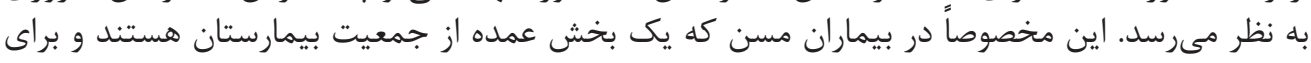

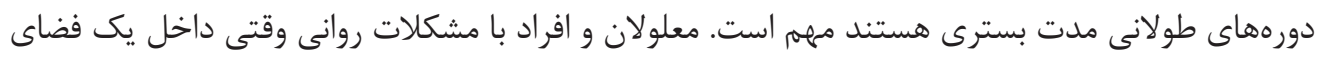

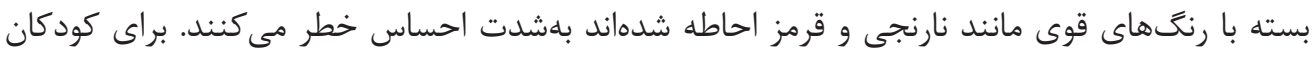

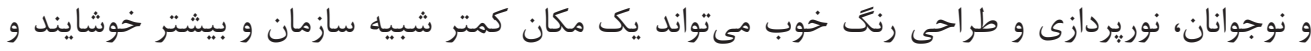

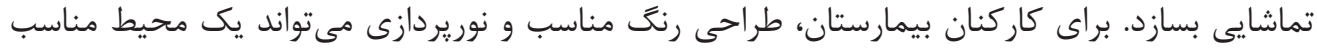

كليد وازهها:

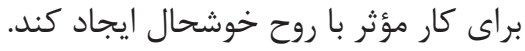

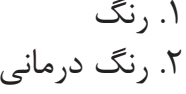

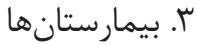

" نويسنده مسئول: بِيرحسين كوليوند

آدرس الكترونيكى: peirhossein@yahoo.com 


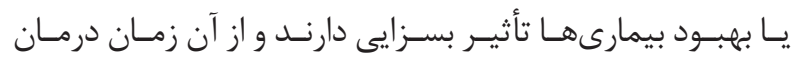

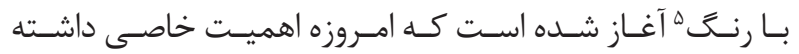

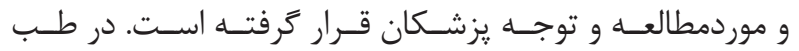

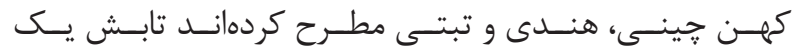

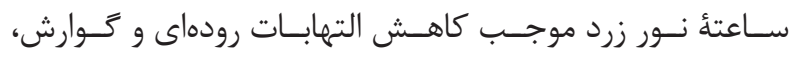

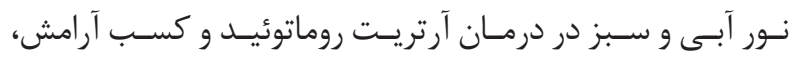

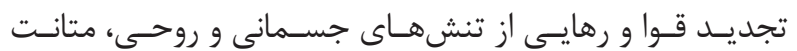

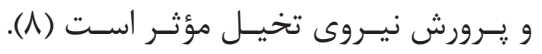

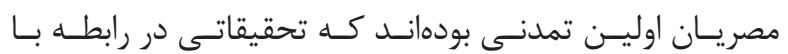

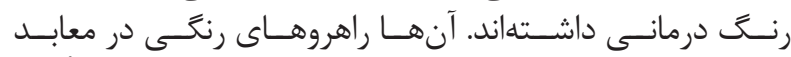

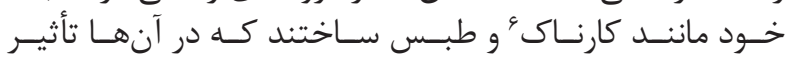

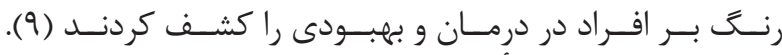

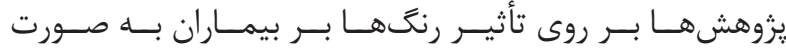

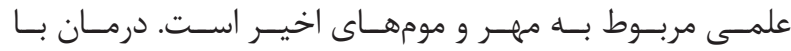

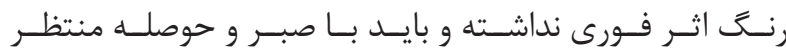

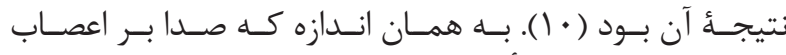

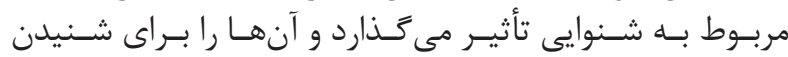

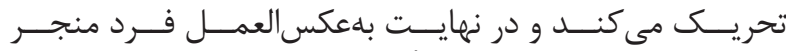

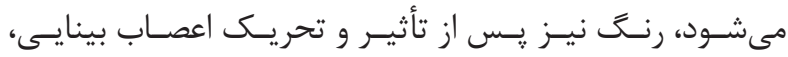

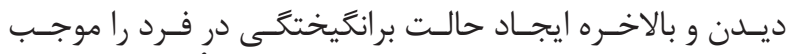

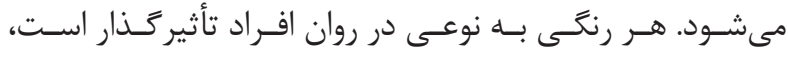

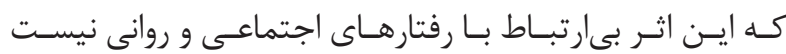

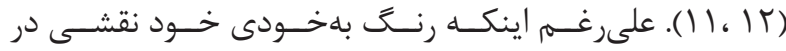

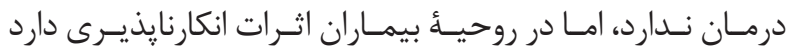

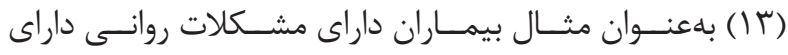

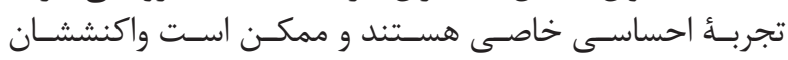

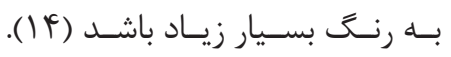

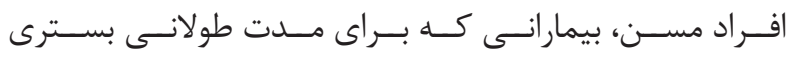

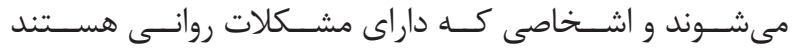

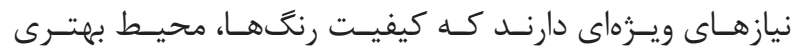

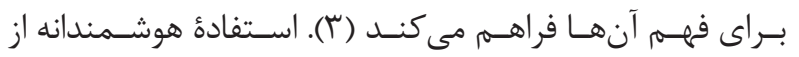

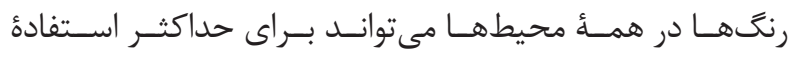

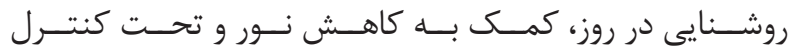

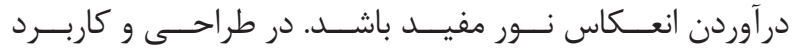

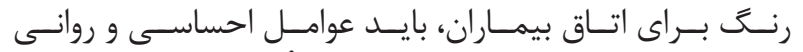

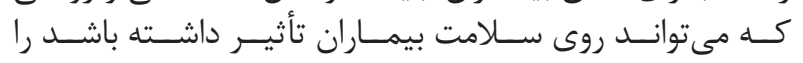

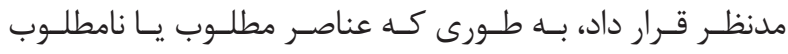

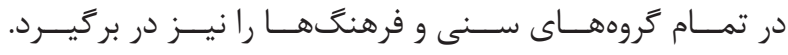

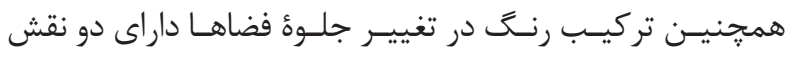

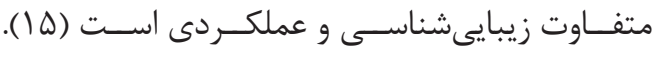

$$
\text { مكانيسم عصبى ديد رنغ مانى }
$$

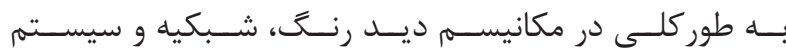

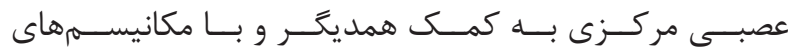

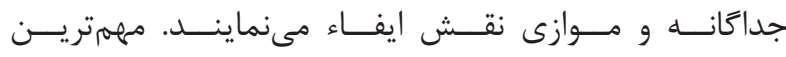

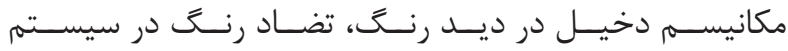

\section{${ }^{1}$ LeGrice}

${ }^{2}$ Saint Augustine

${ }^{3}$ Wassily Kandinsky

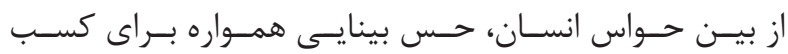

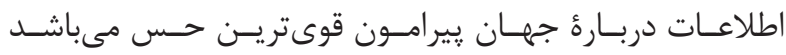

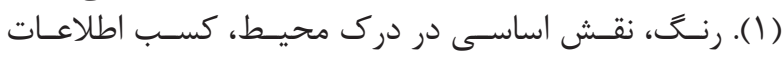

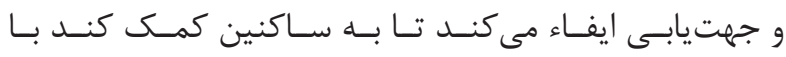

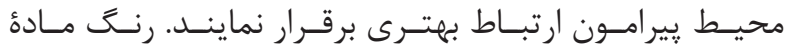

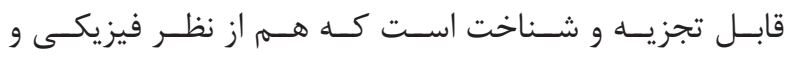

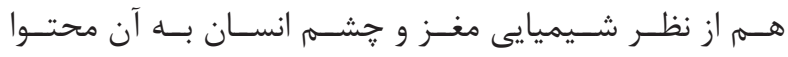

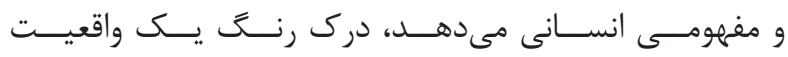

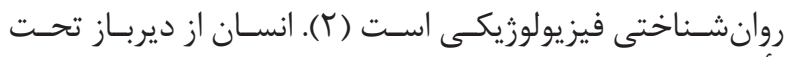

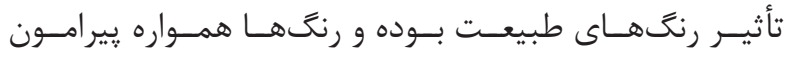

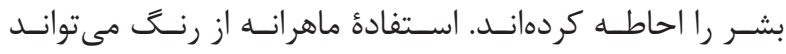

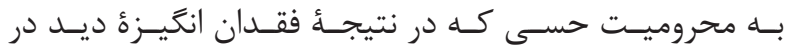

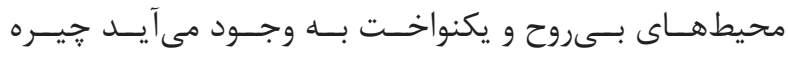

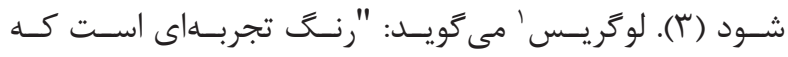

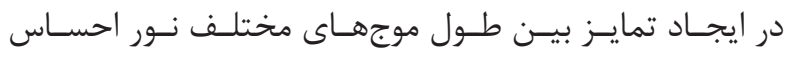

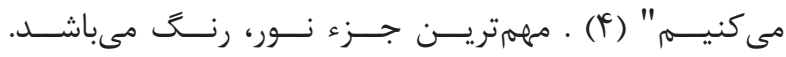

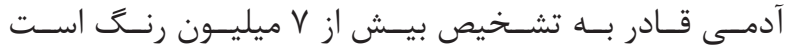

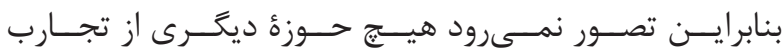

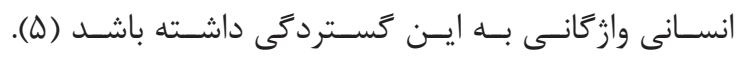

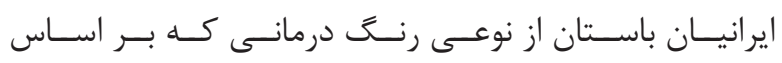

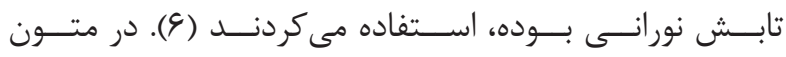

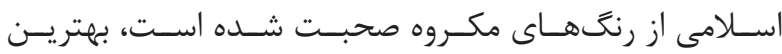

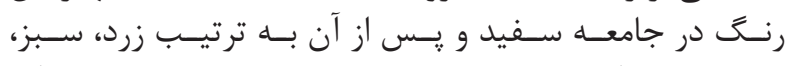

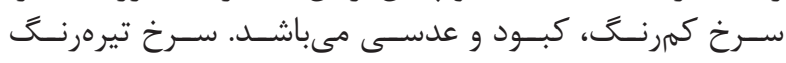

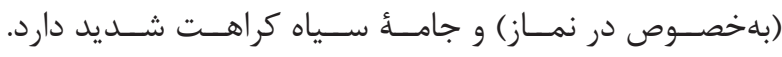

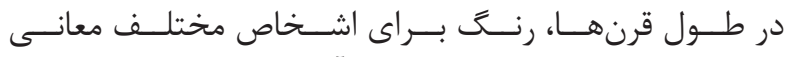

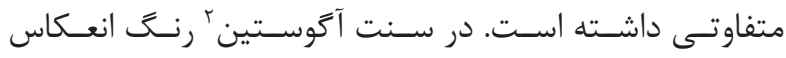

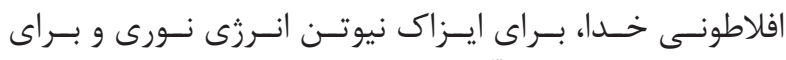

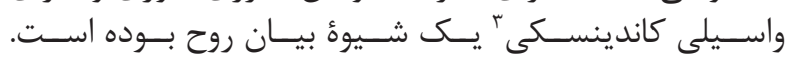

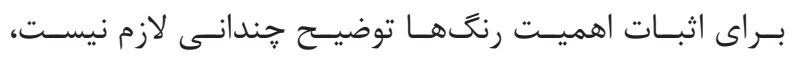

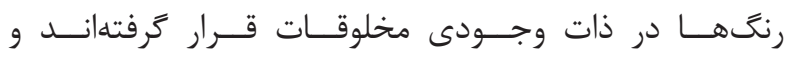

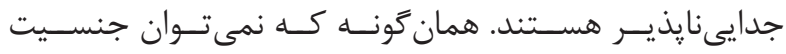

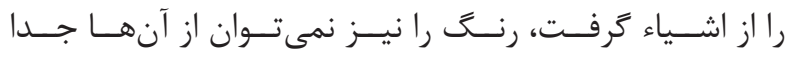

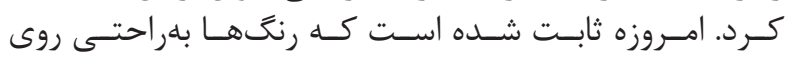

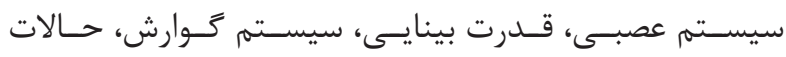

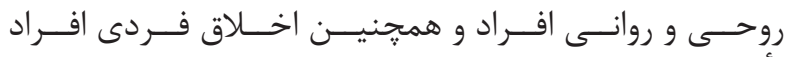

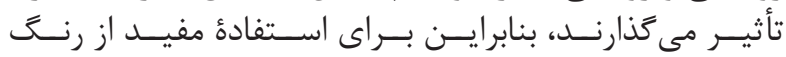

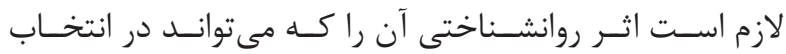

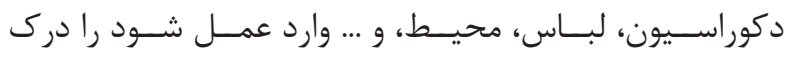

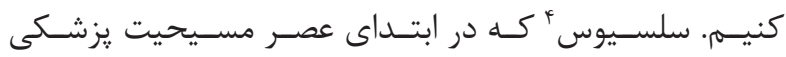

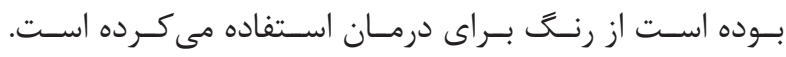

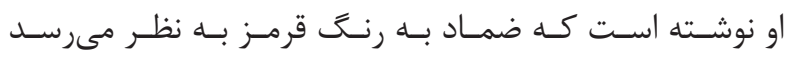

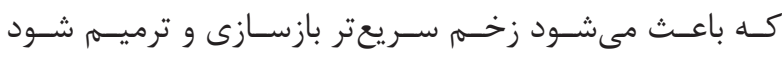

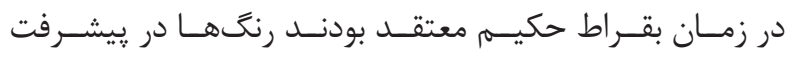

${ }^{4}$ Celsius

${ }^{5}$ Chromotherapy

${ }^{6}$ Karnak 


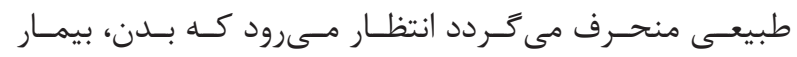

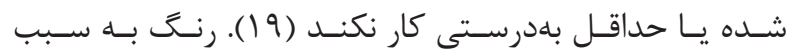

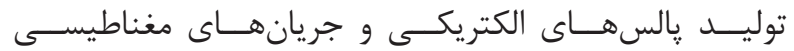

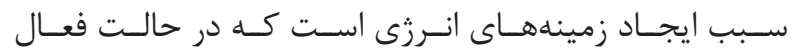

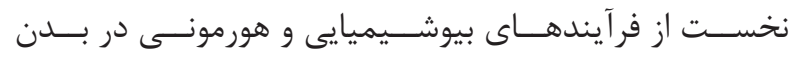

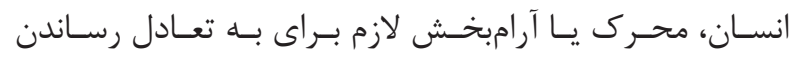

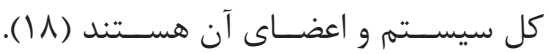

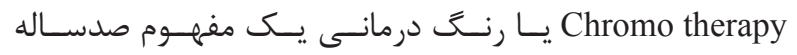

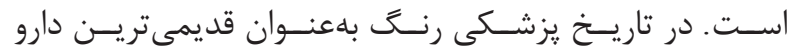

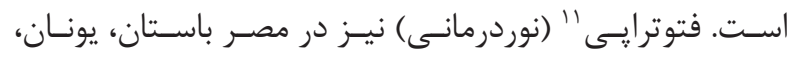

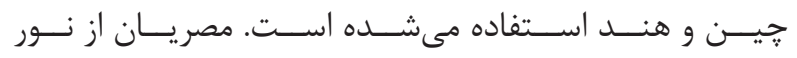

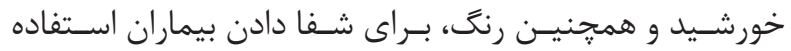

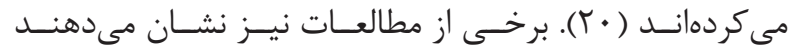

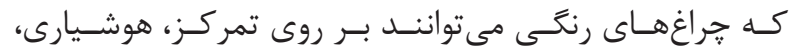

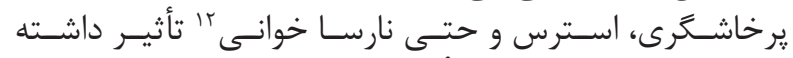

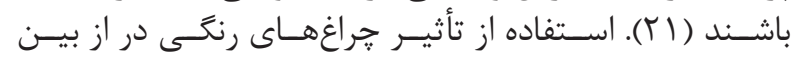

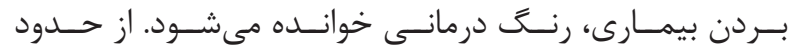

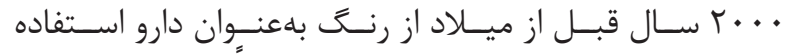

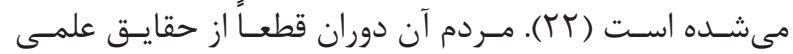

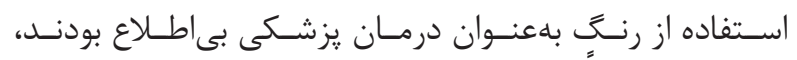

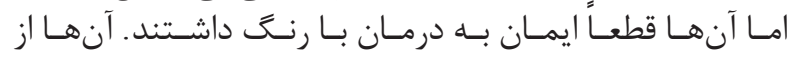

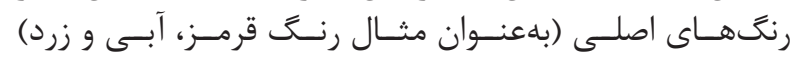

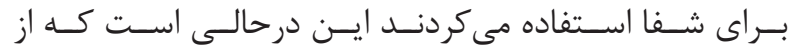

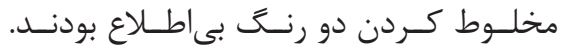

$$
\text { طراحى رنغ محلى }
$$

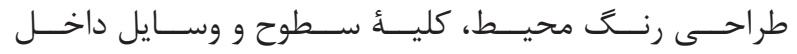

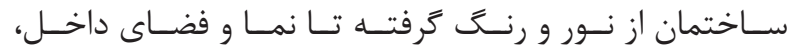

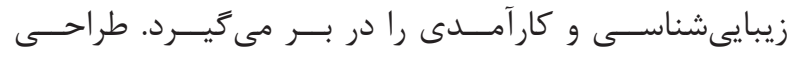

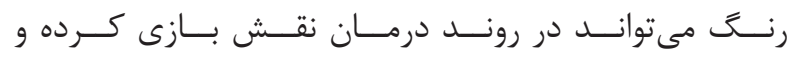

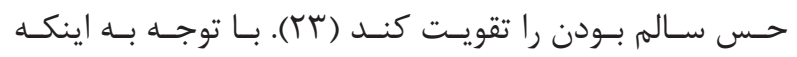

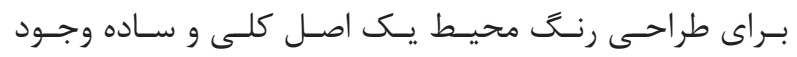

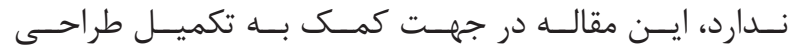

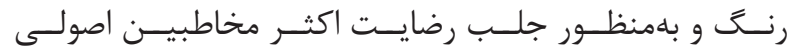

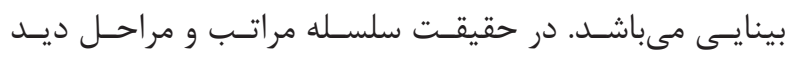

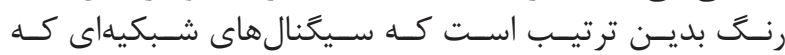

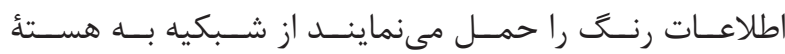

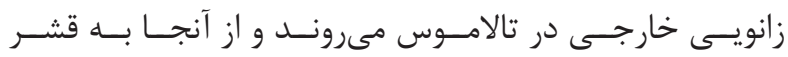

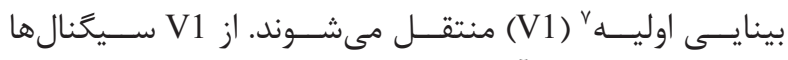

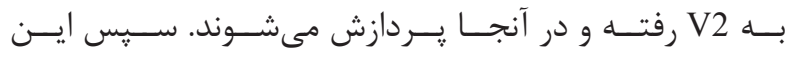

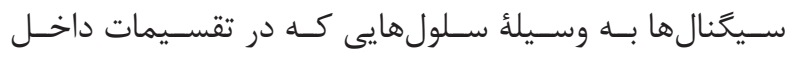

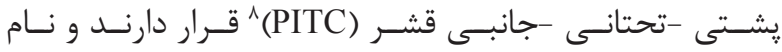

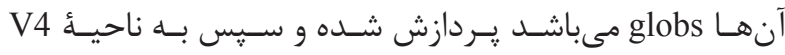

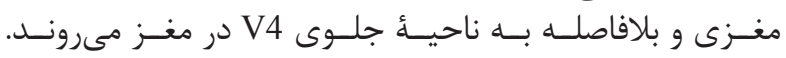

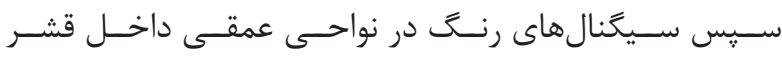

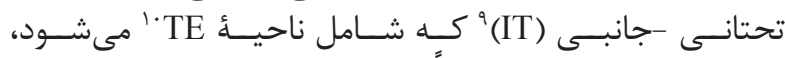

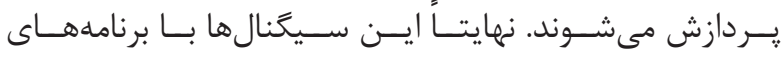

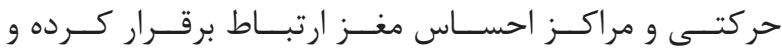

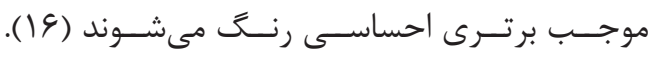

$$
\text { رنت درمانى }
$$

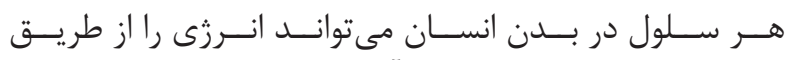

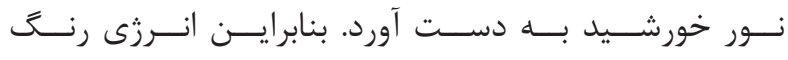

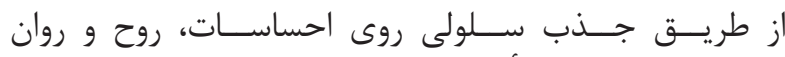

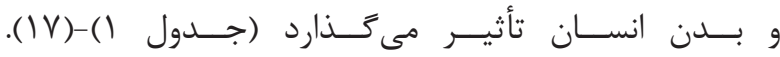

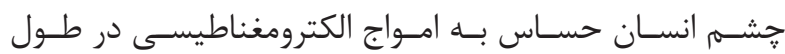

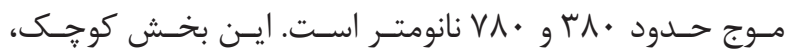

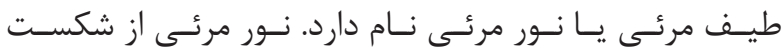

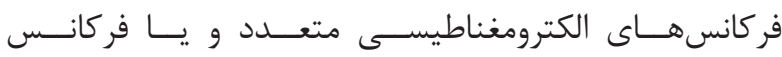

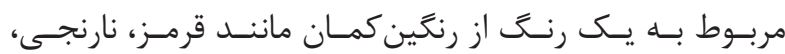

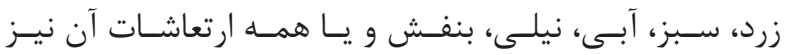

حاصـل مى تـــدد (1) (1).

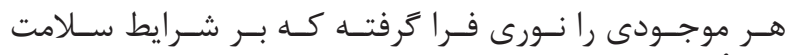

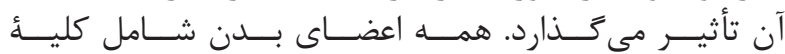

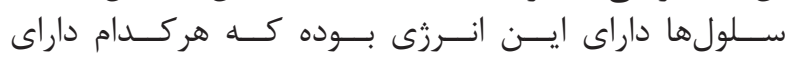

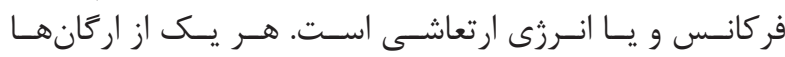

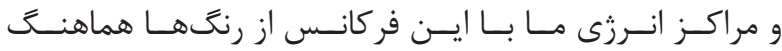

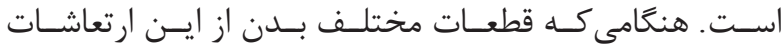

\begin{tabular}{|c|c|}
\hline عملكرو & رنَّ \\
\hline مناسب براى بىخوابى، فشارخون بالا و جهت كاهش حساسيت به درد & آبى \\
\hline آرام كثنده تنش، افزايش هضم، بهبود تعادل توانايىها، حفظ صلح و آرامش درونى و غلبه بر سنكوب & سبز \\
\hline مفيد براى درمان يبوست و افزايش اعتماد به نفس، تحريك سيستم عصبى و سيستم كوارش و بهبود تفكر مثطقى & زرد \\
\hline مفيد براى كاهش اختلالات اعتياد و ميكُرن، بهبود عملكرد سيستم عصبى، لثفاوى و قلبى و عروقى، حفظ تعادل بتاسيسم & بنفش \\
\hline مفيد براى رفع مشكلات مفصلى و واريس & آبي فيروزانى \\
\hline مفيد جهت حفظ انرزىى، افزايش اشتها، كمك به جذب كلسيم، كمك به بهبودى و حفظ سلامت بدن & نارنجى \\
\hline مفيد براى غلبه بر حس كسالت، بهبود تمايلات جثسى، تحريك سيستم عصبى، افزايش آدرنالين و كردش خون، حفظ & قرمز \\
\hline
\end{tabular}

جدول ا- تأثير رنگها بر درمان و عملكردهاى بدن (IV).

${ }^{7}$ Primary visual cortex

${ }^{8}$ Posterior inferior temporal cortex

${ }^{9}$ Inferior temporal
${ }^{10}$ Inferotemporal cortex

${ }^{11}$ Phototherapy

${ }^{12}$ Dyslexia 


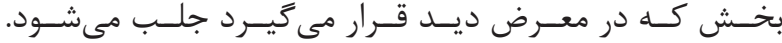

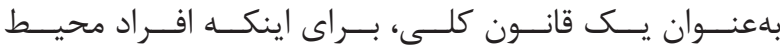

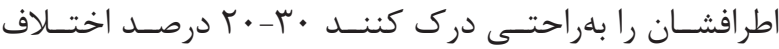

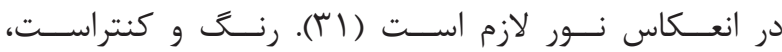

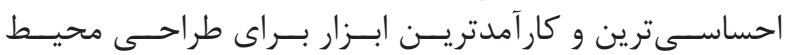

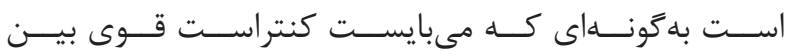

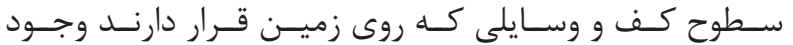

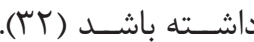

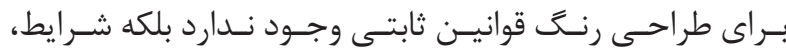

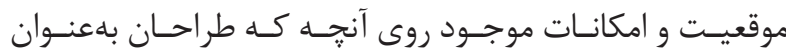

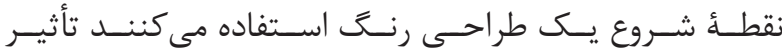

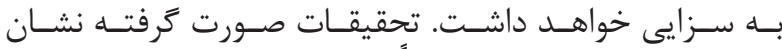

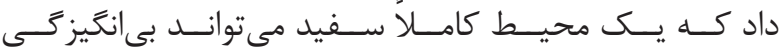

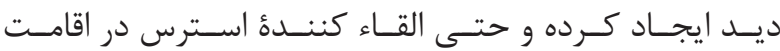

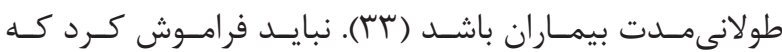

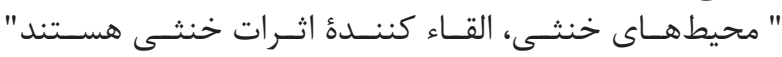

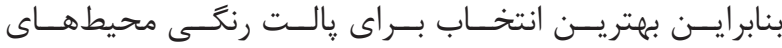

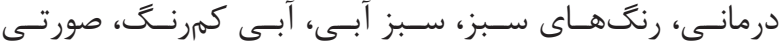

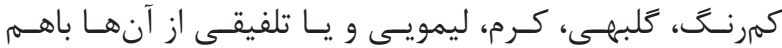

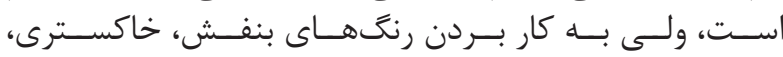

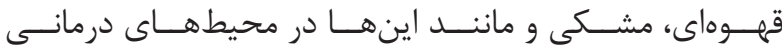

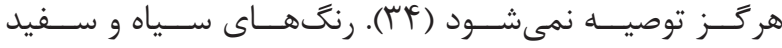

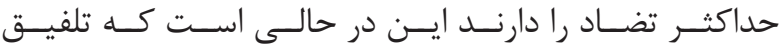

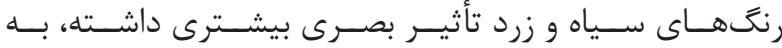

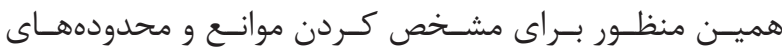

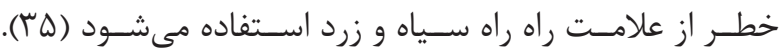

$$
\text { توصيههاى كلى در مورد رنتَها }
$$

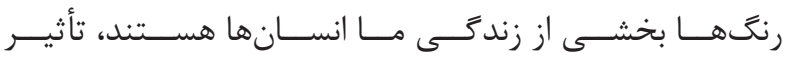

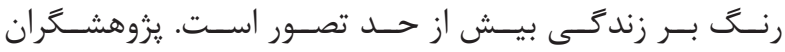

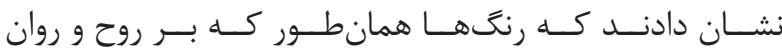

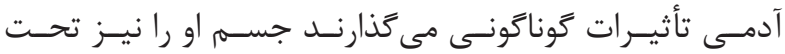

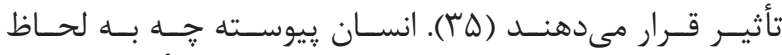

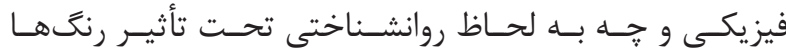

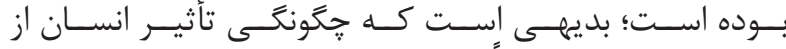

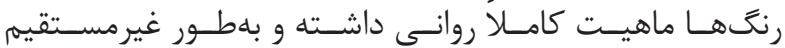

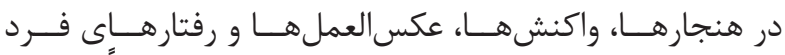

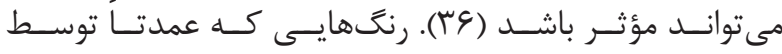

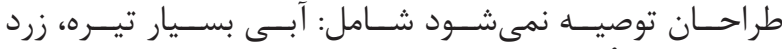

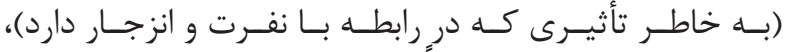

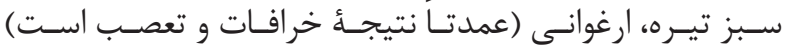

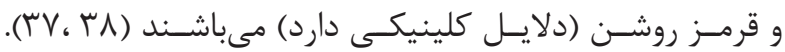

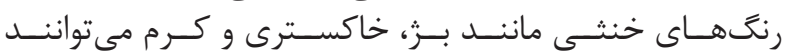

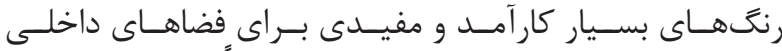

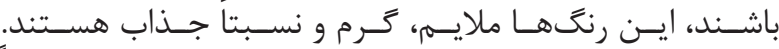

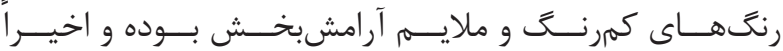

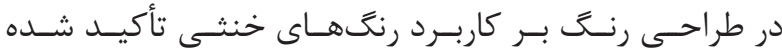

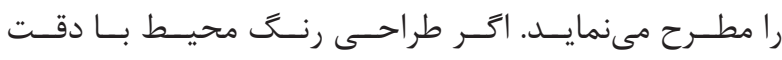

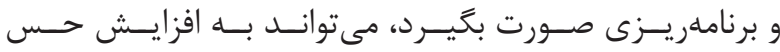

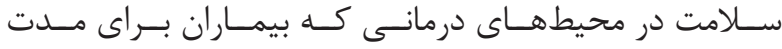

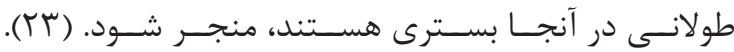

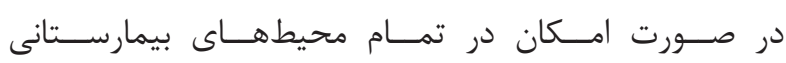

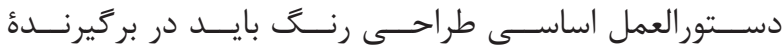

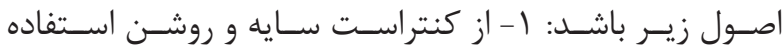

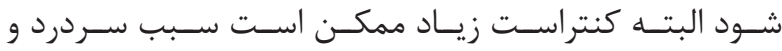

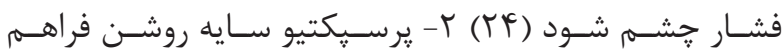

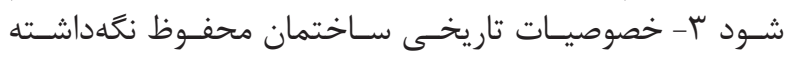

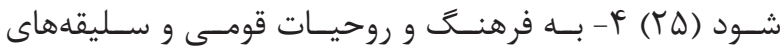

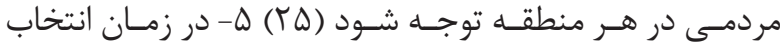

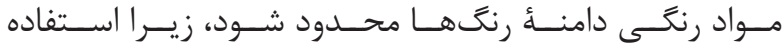

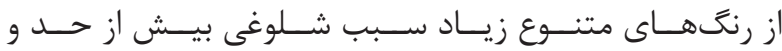

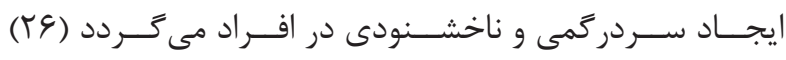

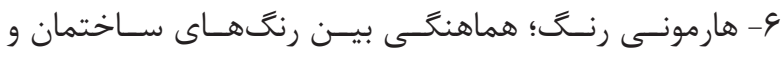

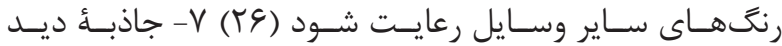

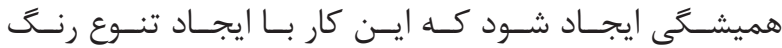

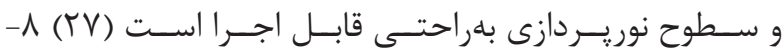

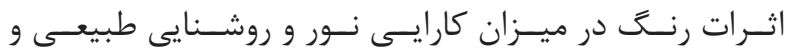

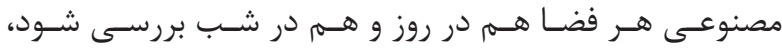

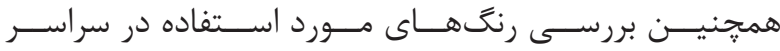

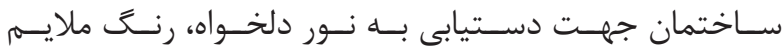

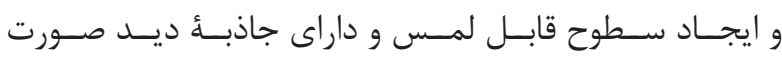

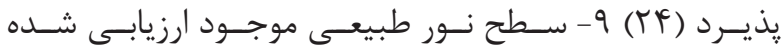

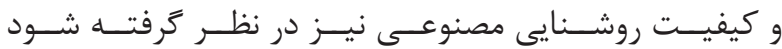

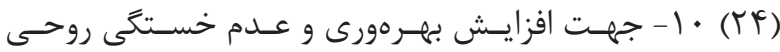

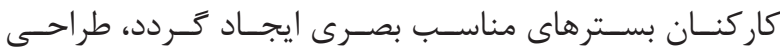

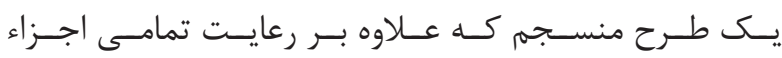

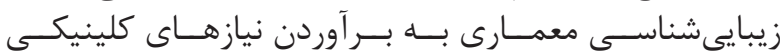

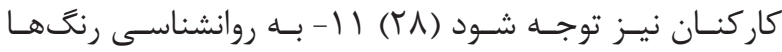

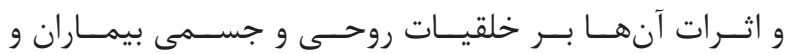

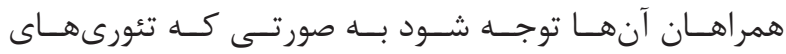

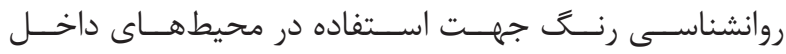

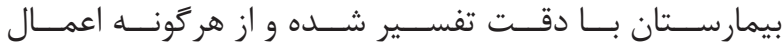

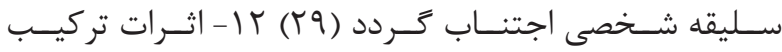

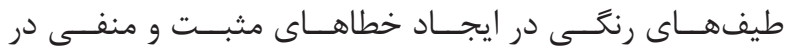

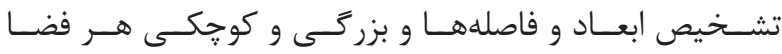

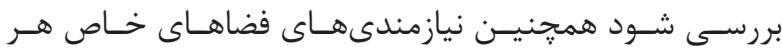

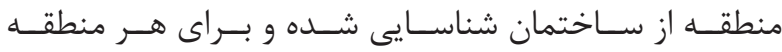

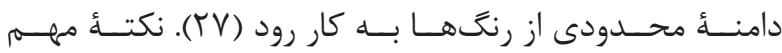

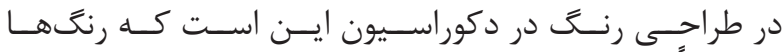

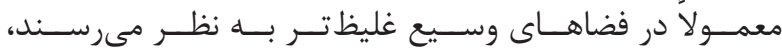

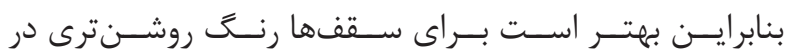

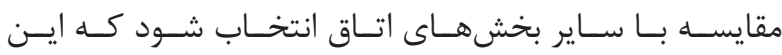

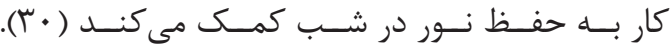

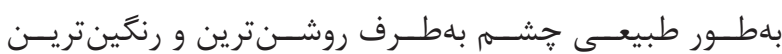




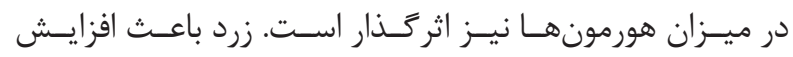

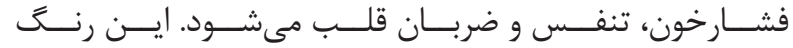

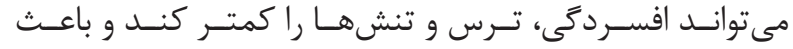

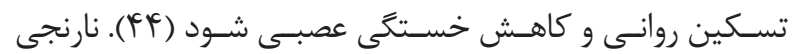

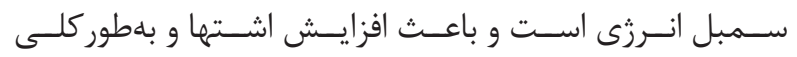

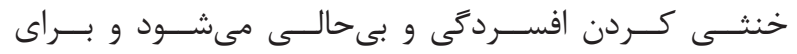

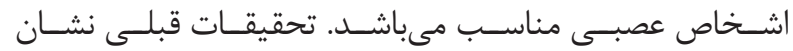

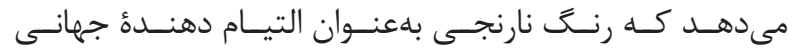

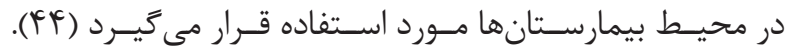

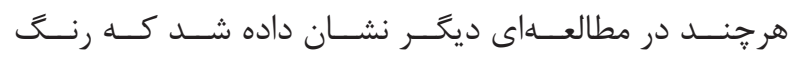

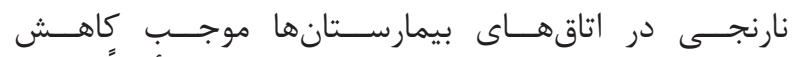

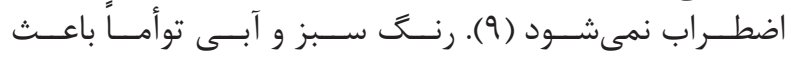

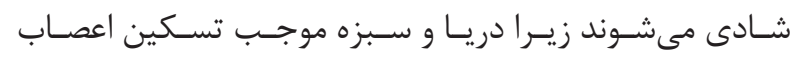

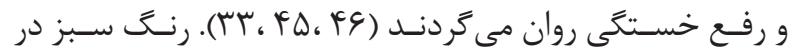

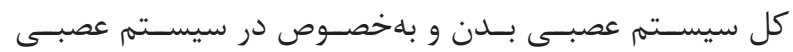

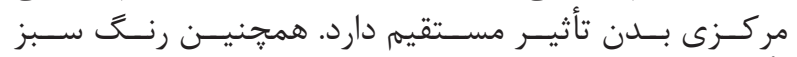

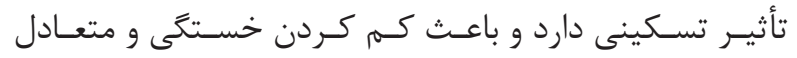

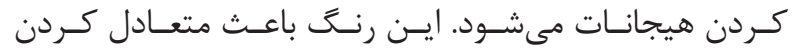

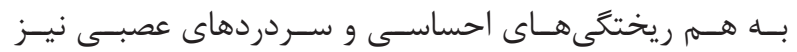

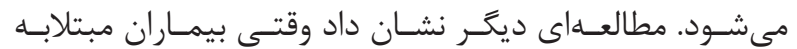

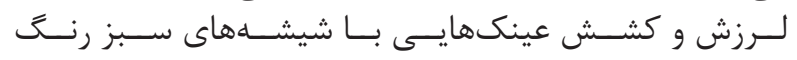

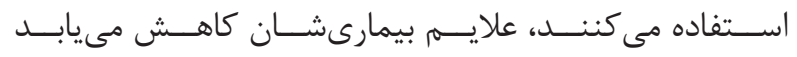

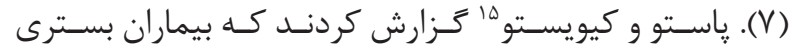

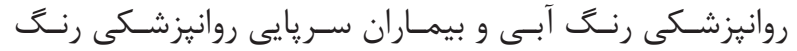

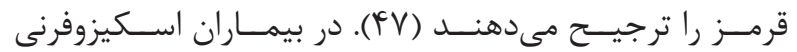

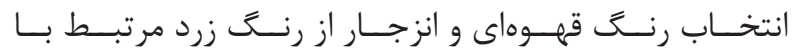

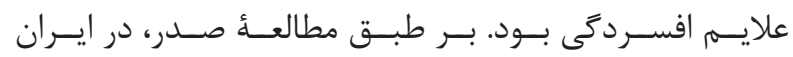

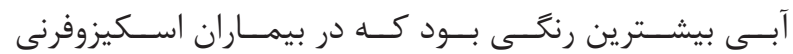

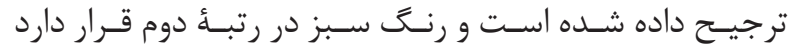

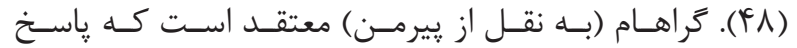

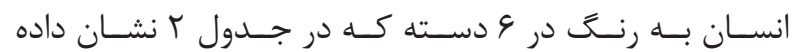

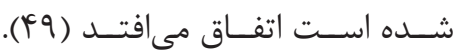

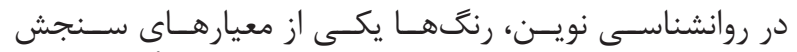

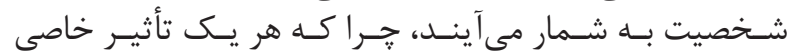

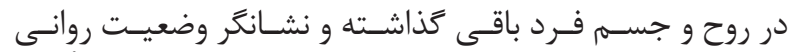

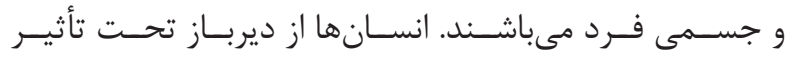

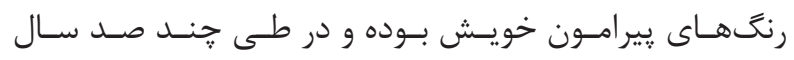

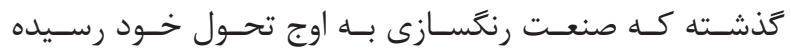

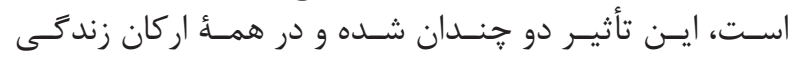

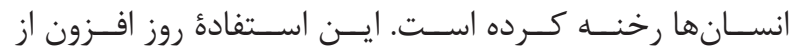

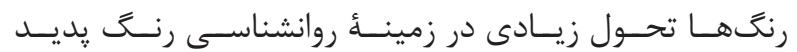

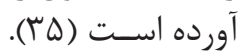

مبانى رنتَ

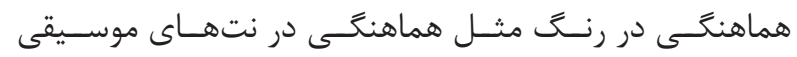

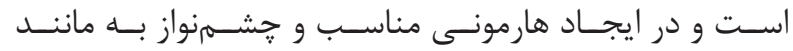

${ }^{13}$ Max Lüscher

${ }^{14}$ Vernolia

${ }^{15}$ Pasto and Kivisto

\section{توجه به تكنيكهاى ايجاد خطا در ديد}

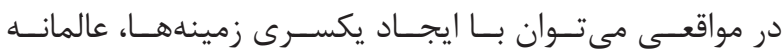

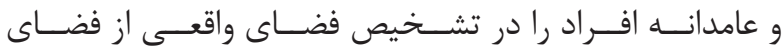

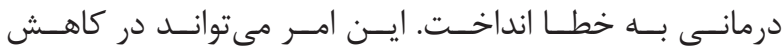

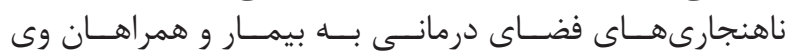

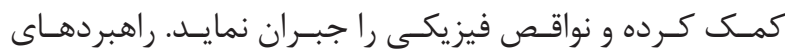

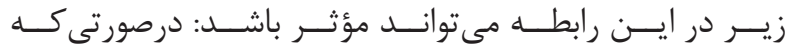

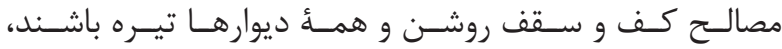

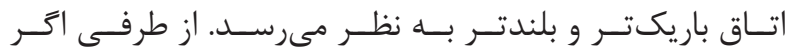

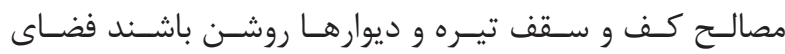

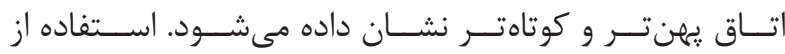

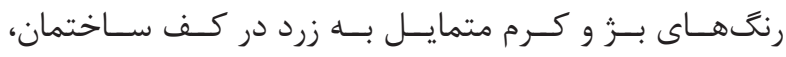

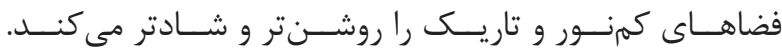

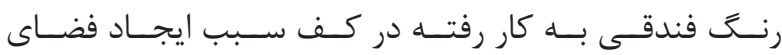

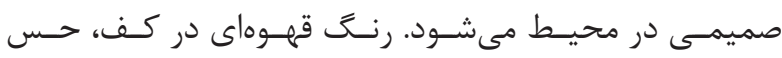

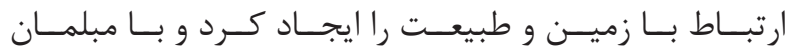

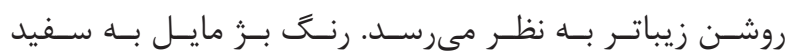

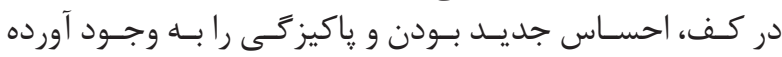

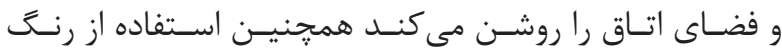

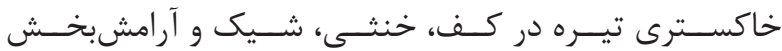

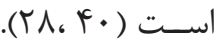

تأثير روانى رنغَان

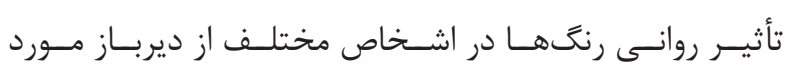

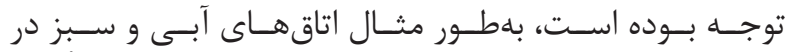

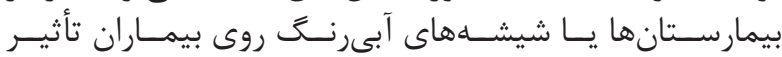

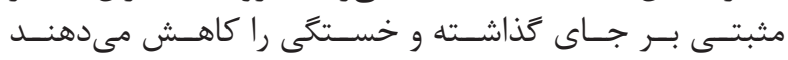

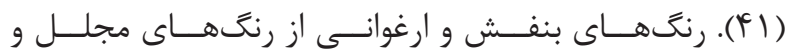

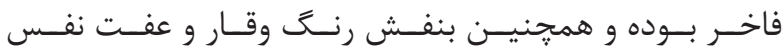

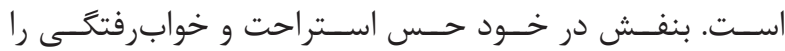

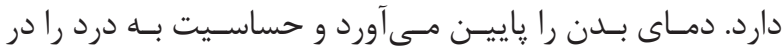

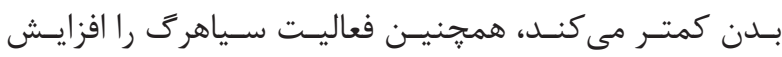

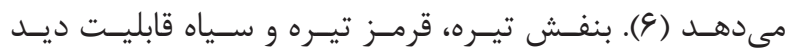

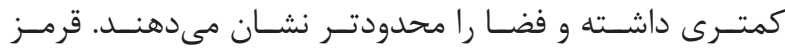

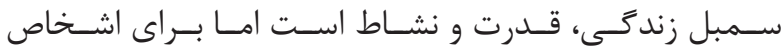

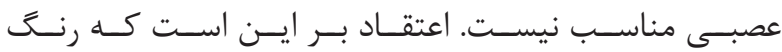

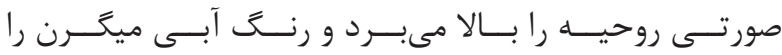

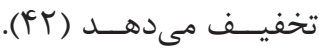

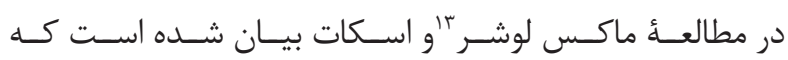

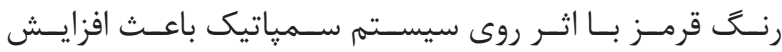

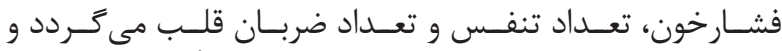

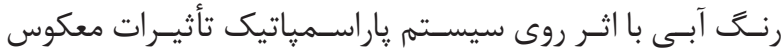

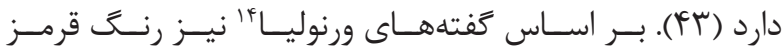

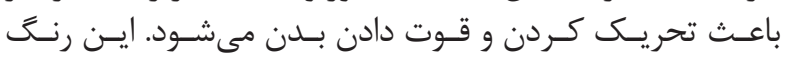

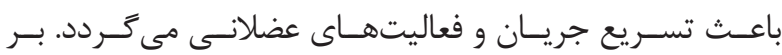

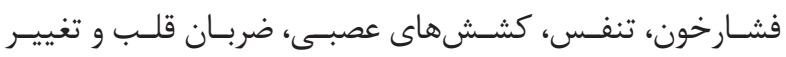


جدول r- تأثير رنكَها بر بيماران (9))

\begin{tabular}{|c|c|}
\hline اكتيايش در رشد رشارخون، ضربان قلب، سيستم عصبى خودكار بدن، فعاليت هورمونها، سرعت & \\
\hline 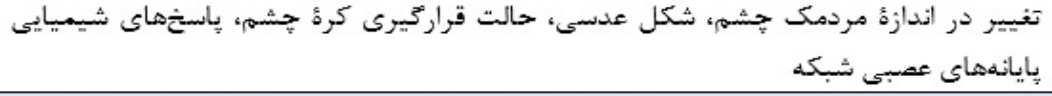 & در جشمهـا \\
\hline 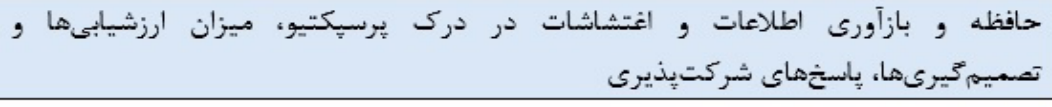 & \\
\hline 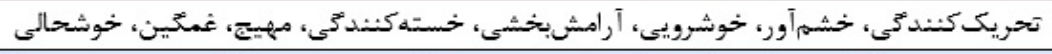 & خلق و خو و حالتهاى روانى \\
\hline 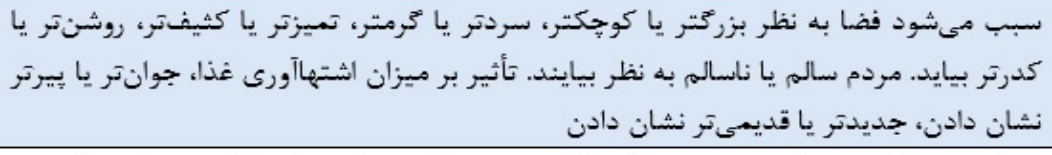 & 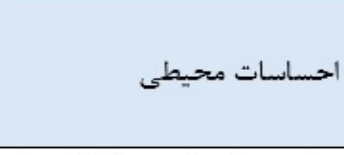 \\
\hline به طبيعت، فناورى، مذهب و سنتثها فرهنتِّى، هنر و علم معمولى ما و يـا غير معمولى ماثي & ميل به وابستئكى و مشاركتهـا \\
\hline
\end{tabular}

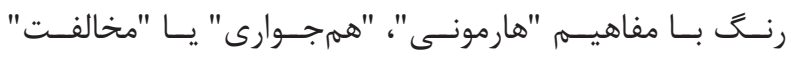

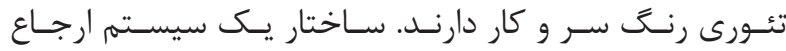

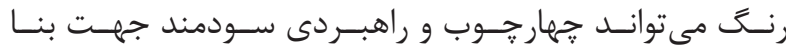

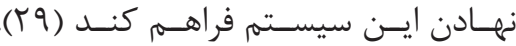

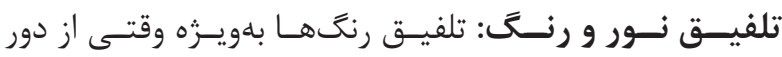

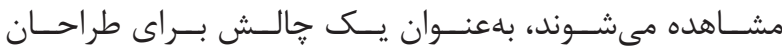

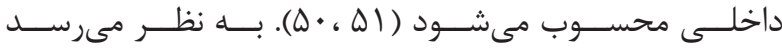

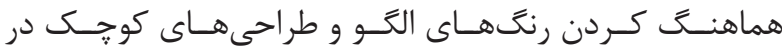

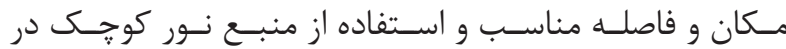

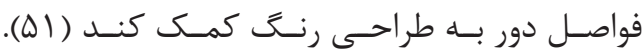

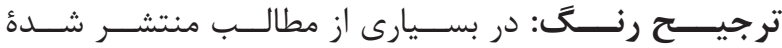

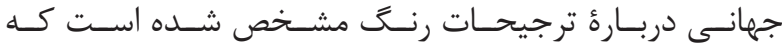

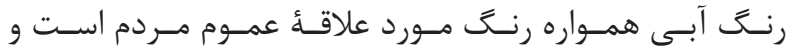

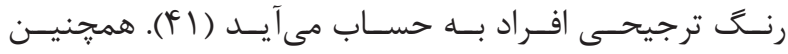

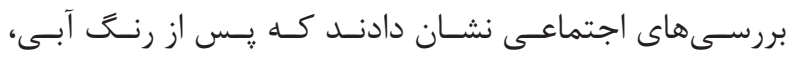

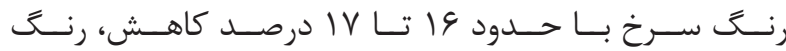

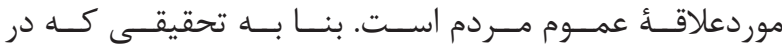

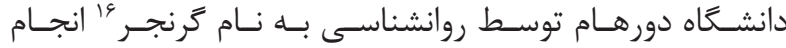

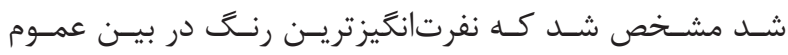

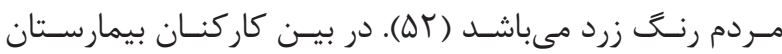

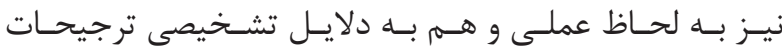

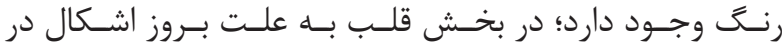

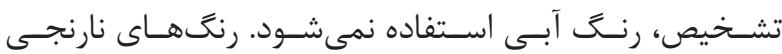

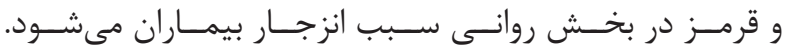

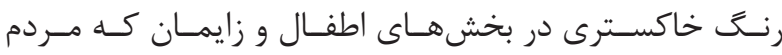

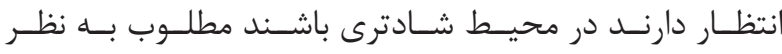

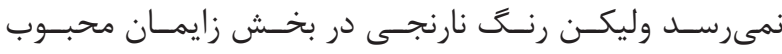

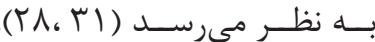

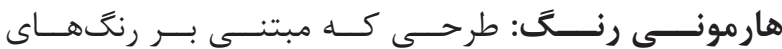

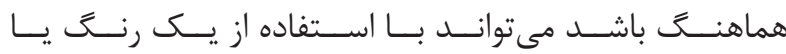

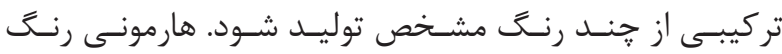

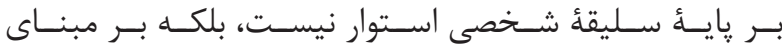

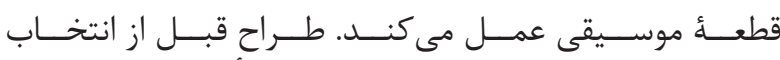

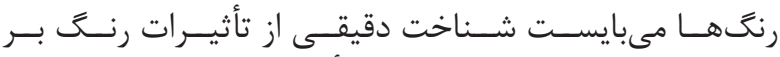

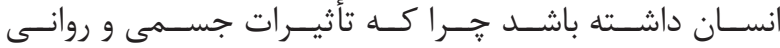

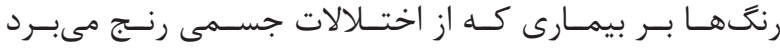

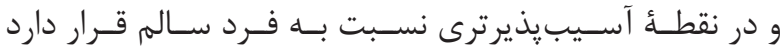

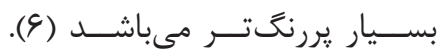

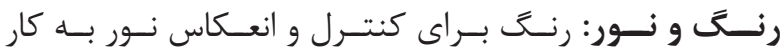

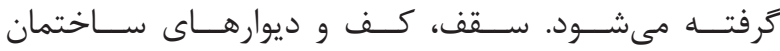

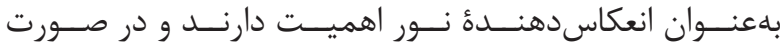

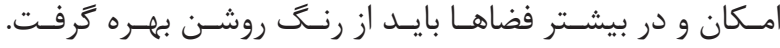

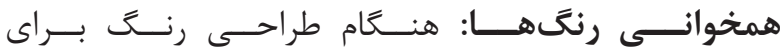

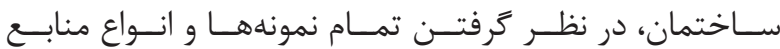

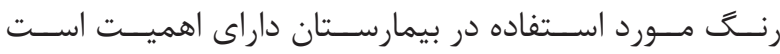

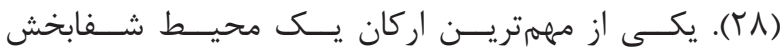

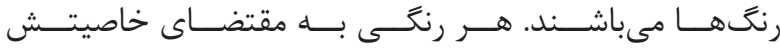

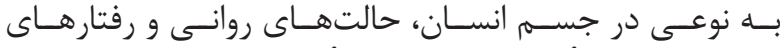

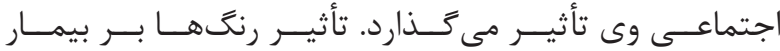

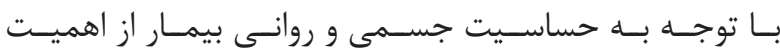

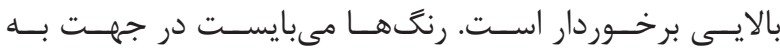

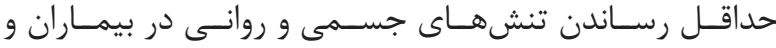

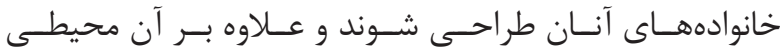

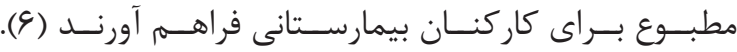

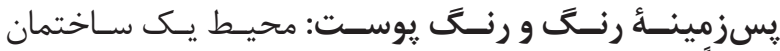

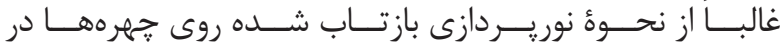

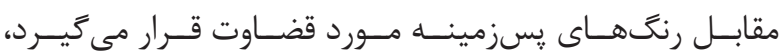

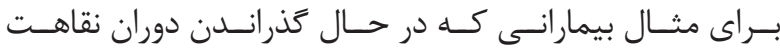

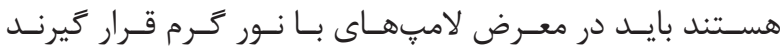

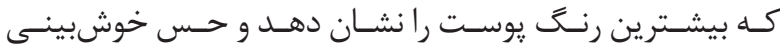

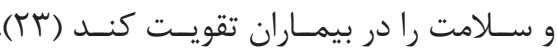

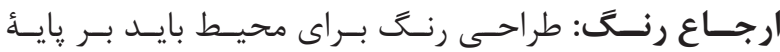

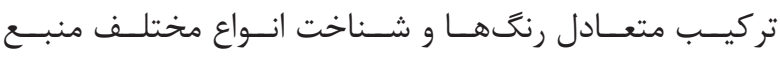

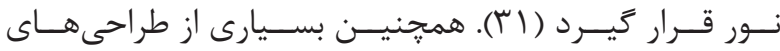




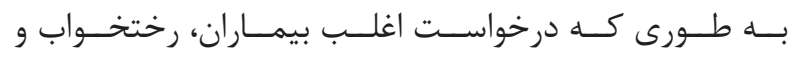

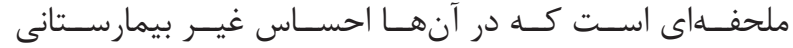

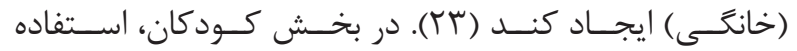

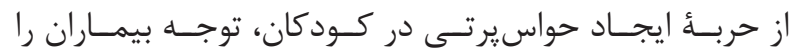

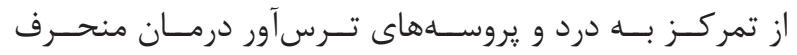

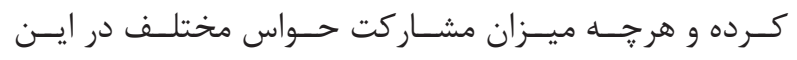

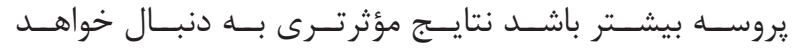

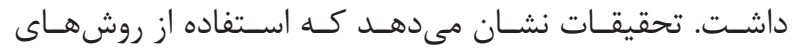

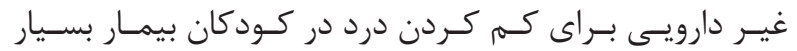

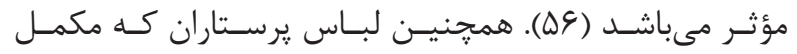

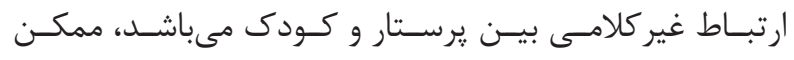

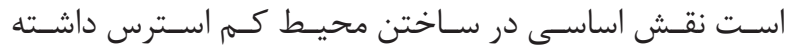

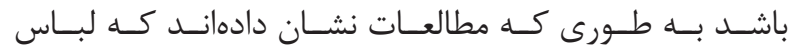

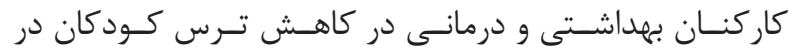

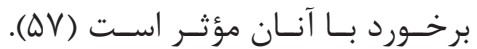

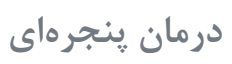

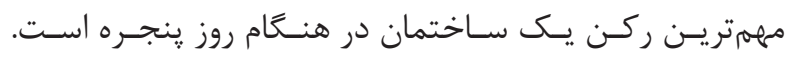

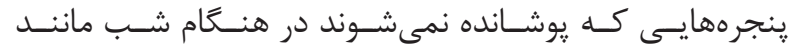

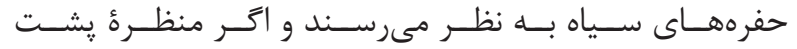

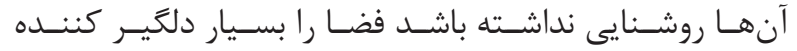

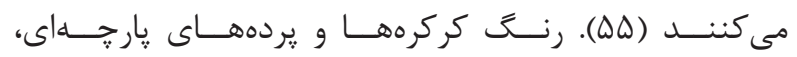

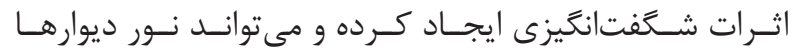

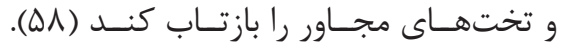

راهبردهاى طراحى رنَّ در محيط بيمارستان

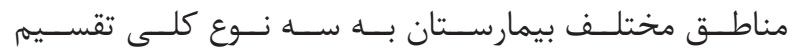

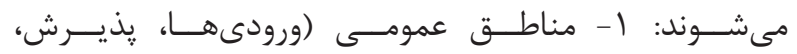

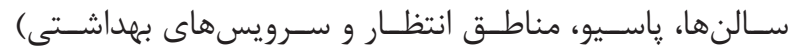

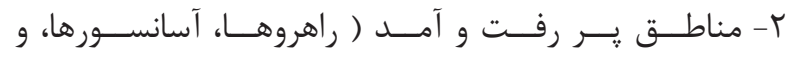

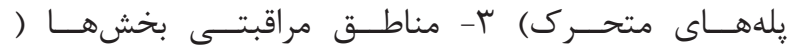

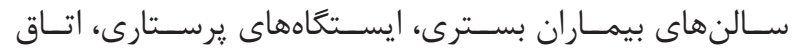
كاركنــان، ياويونهـان.

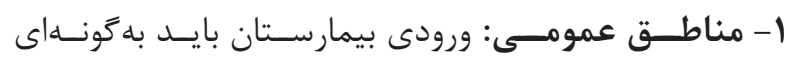

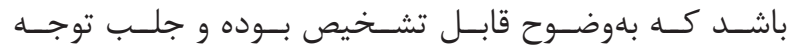

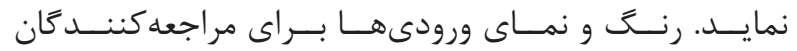

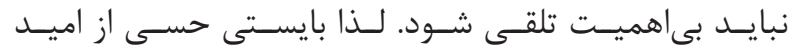

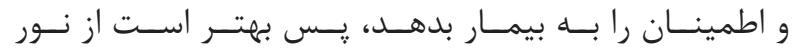

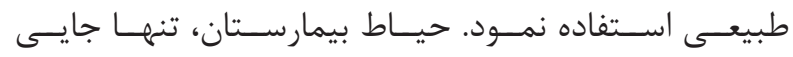

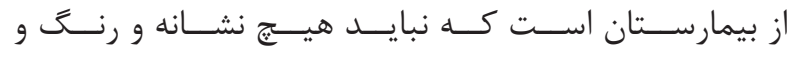

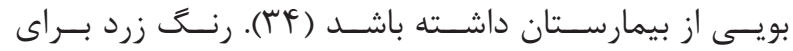

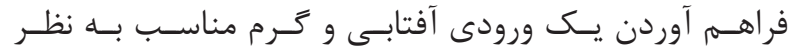

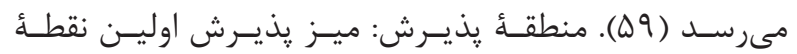

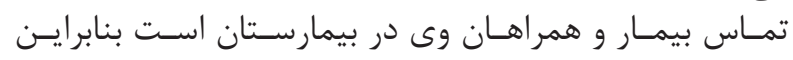

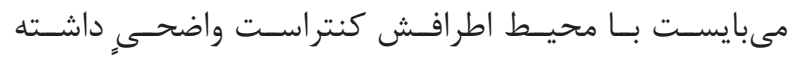

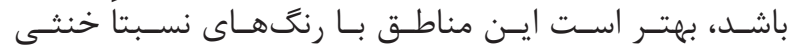

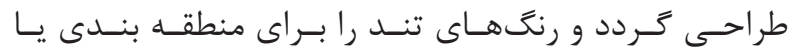

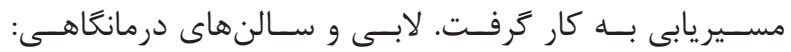

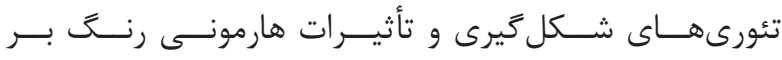

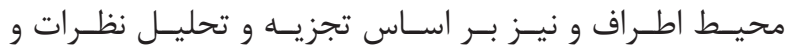

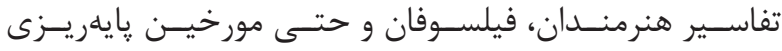

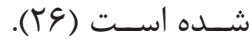

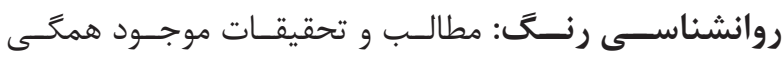

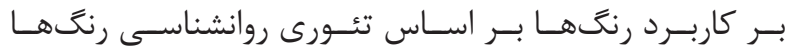

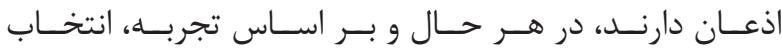

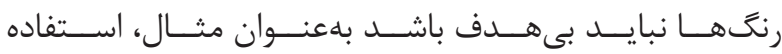

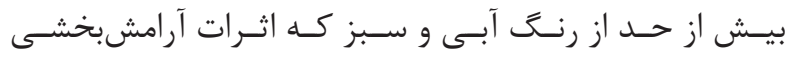

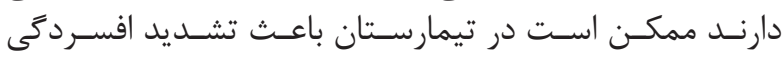

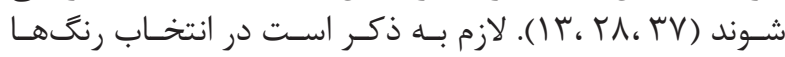

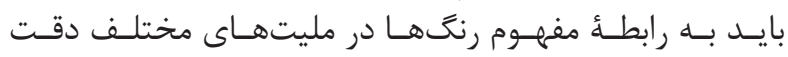

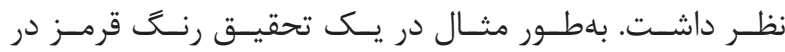

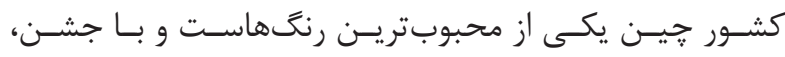

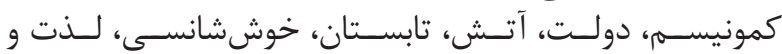

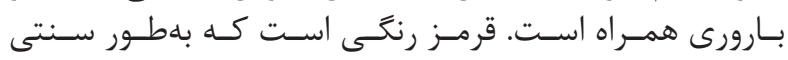

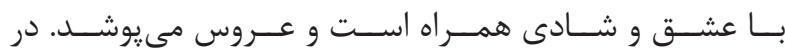

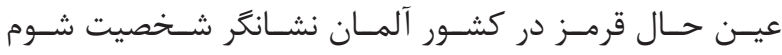

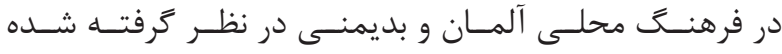

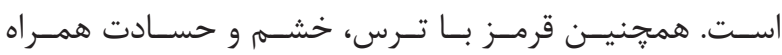

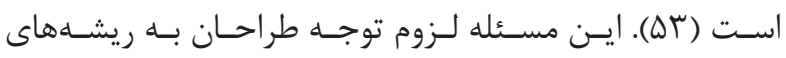

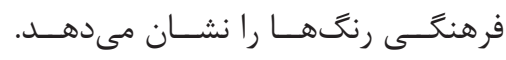

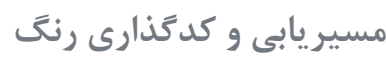

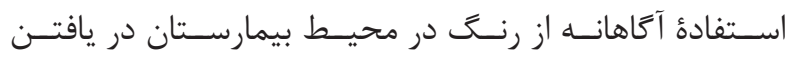

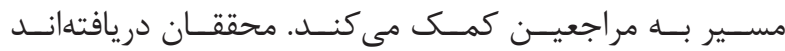

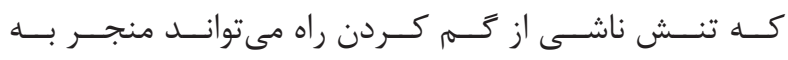

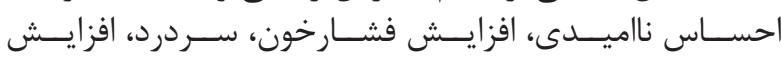

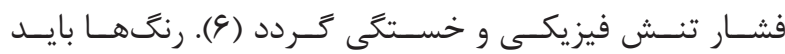

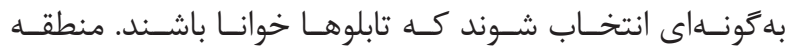

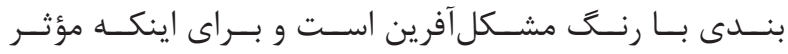

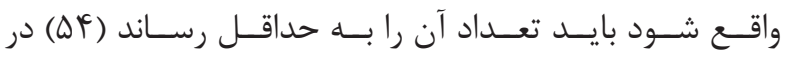

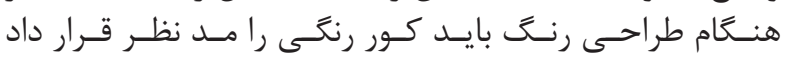

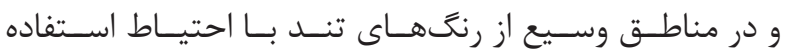

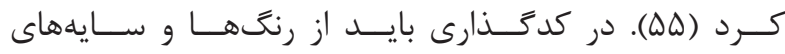

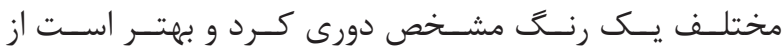

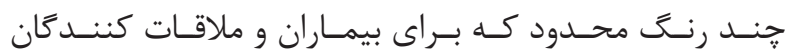

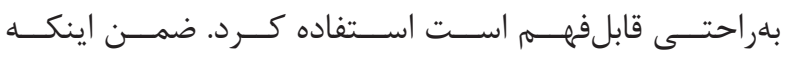

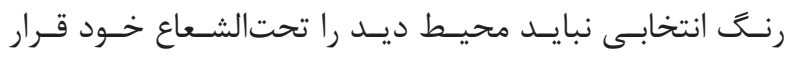

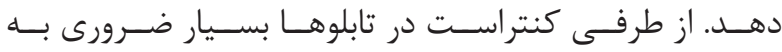

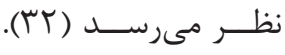
رنتَ و وسايل

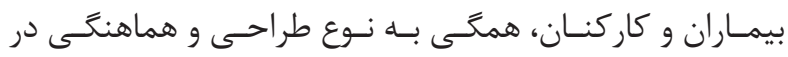

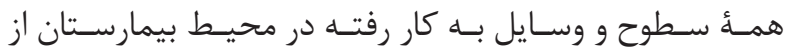

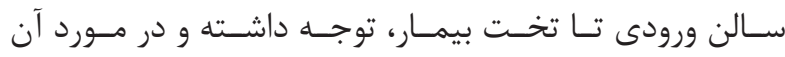

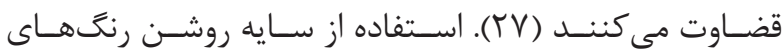

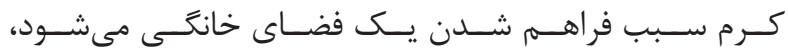




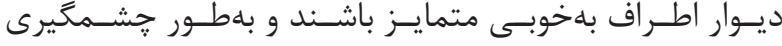

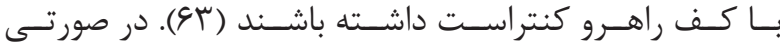

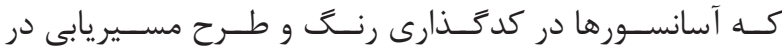

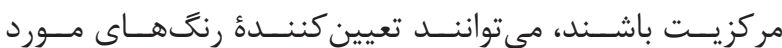

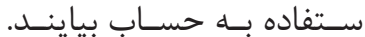

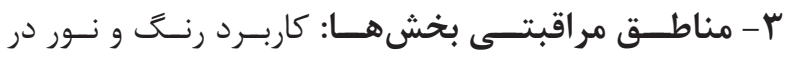

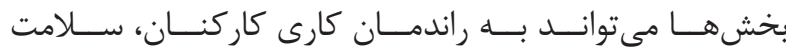

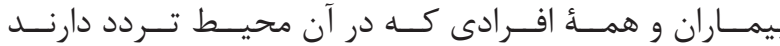

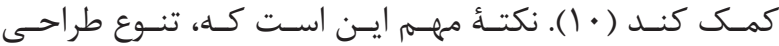

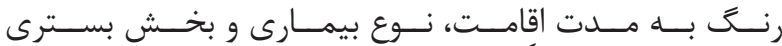

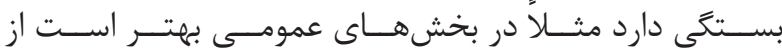

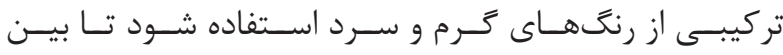

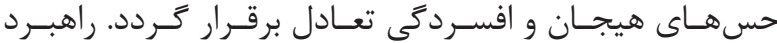

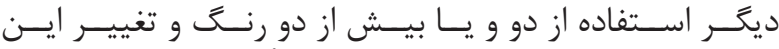

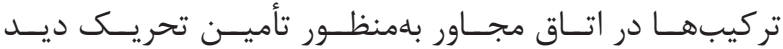

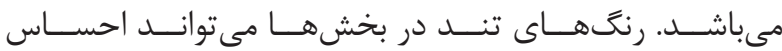

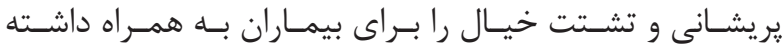

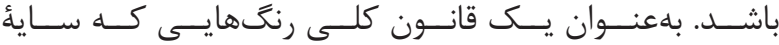

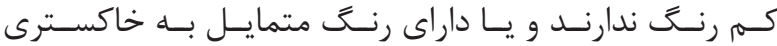

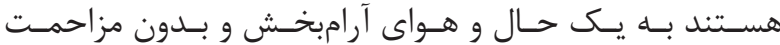

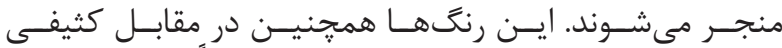

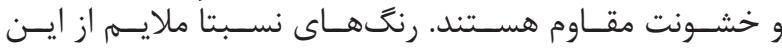

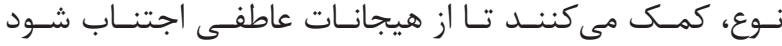

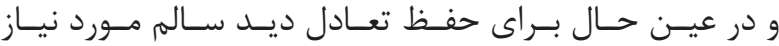

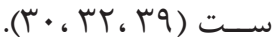

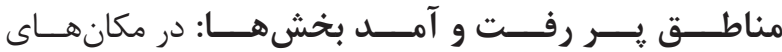

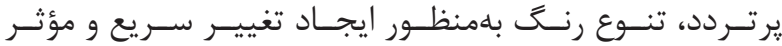

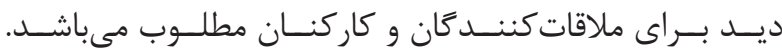

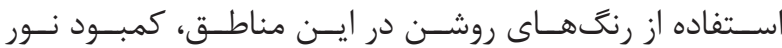

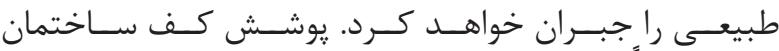

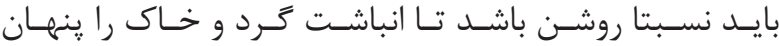

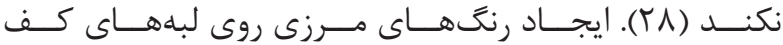

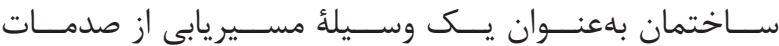

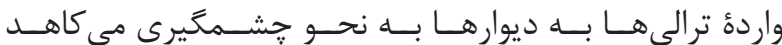

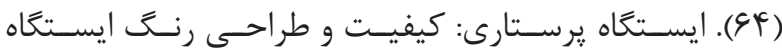

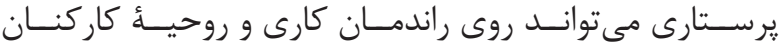

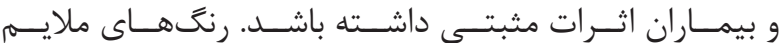

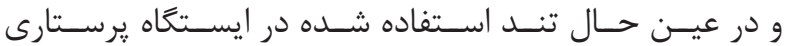

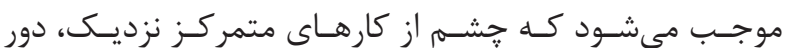

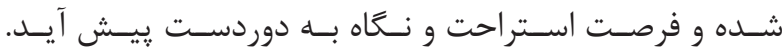

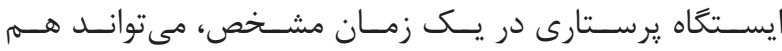

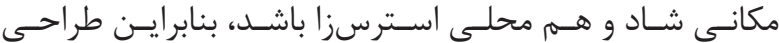

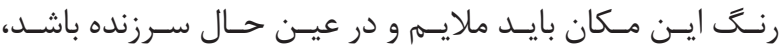

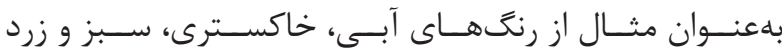

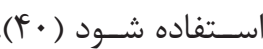

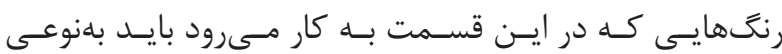

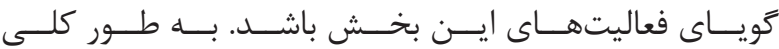

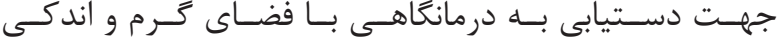

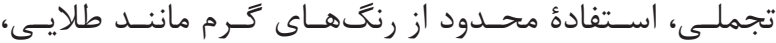

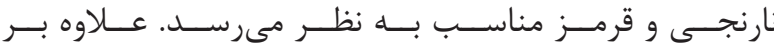

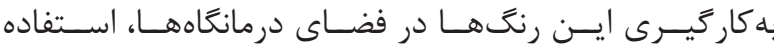

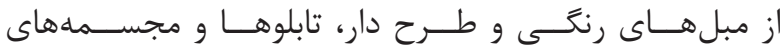

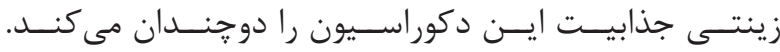

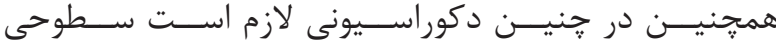

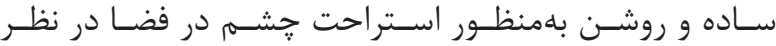

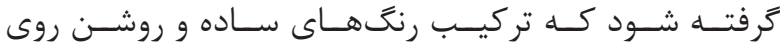

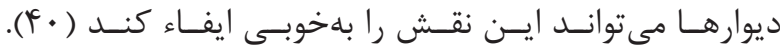

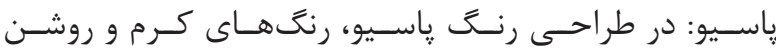

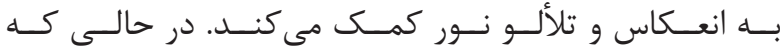

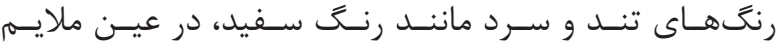

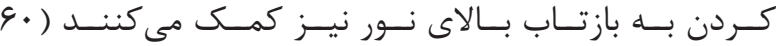

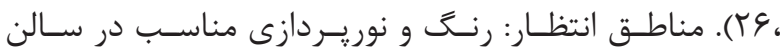

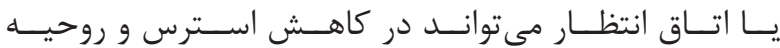

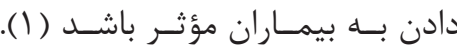

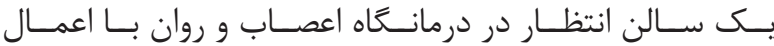

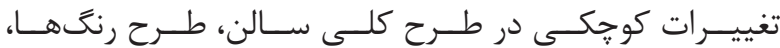

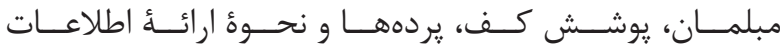

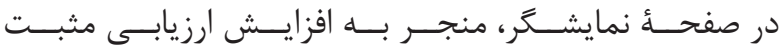

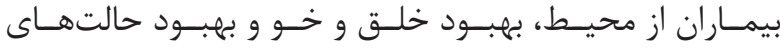

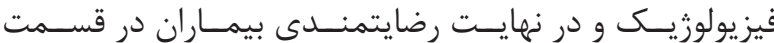

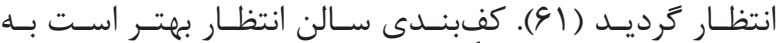

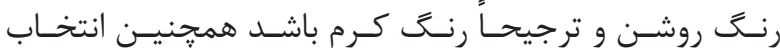

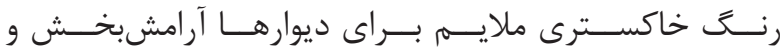

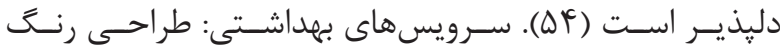

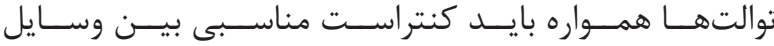
بهداشـتى و ديوارهــا ايجــاد كنــد.

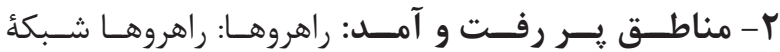

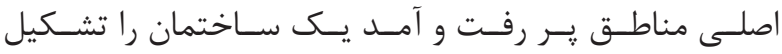

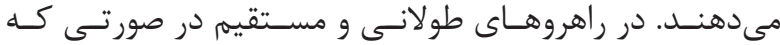

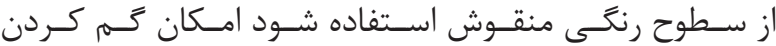

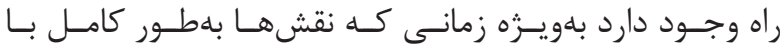

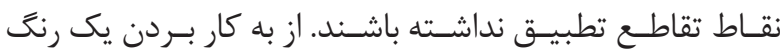

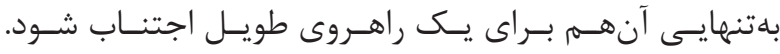

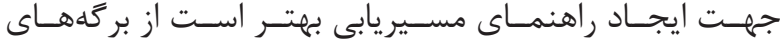

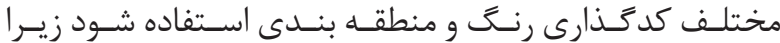

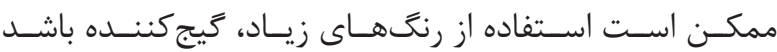

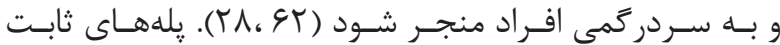

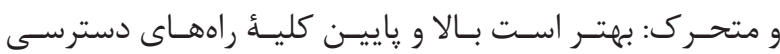

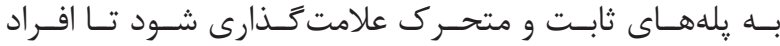

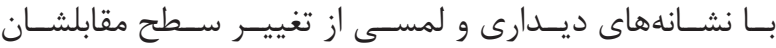

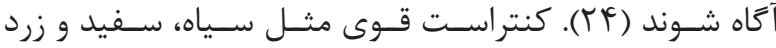

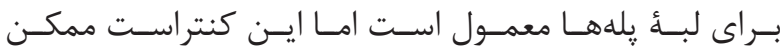

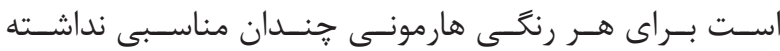

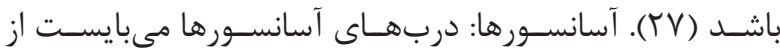




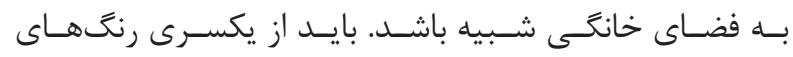

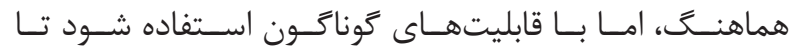

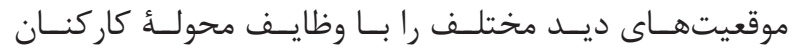

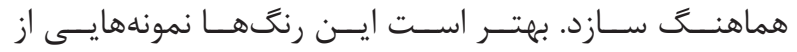

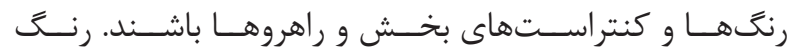

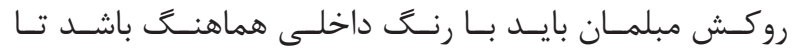

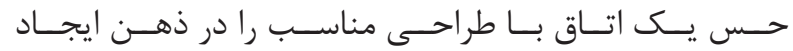

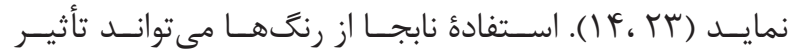

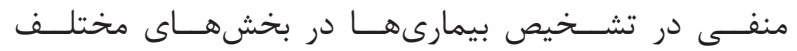

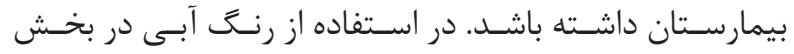

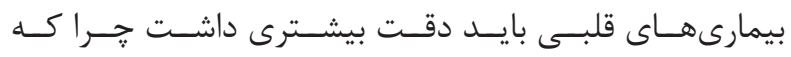

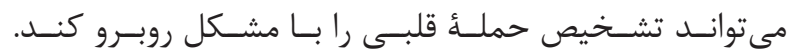

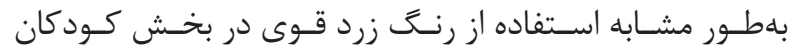

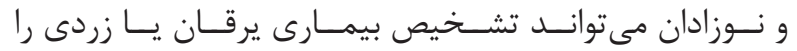

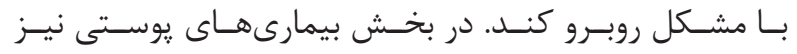

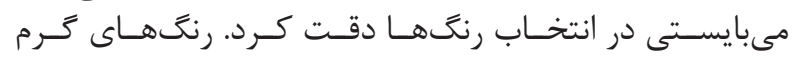

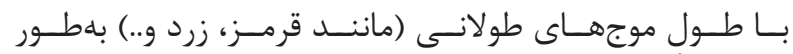

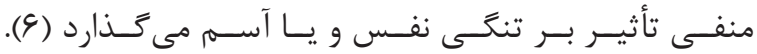
نتيجه گيرى

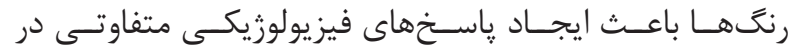

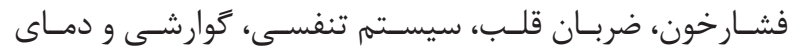

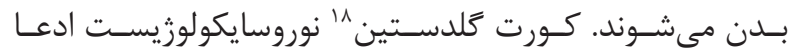

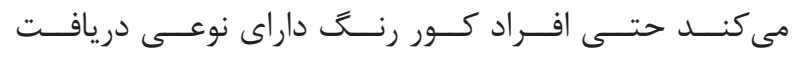

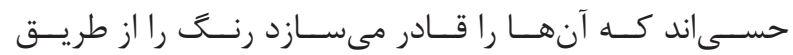

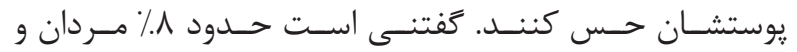

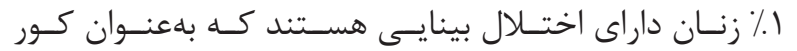

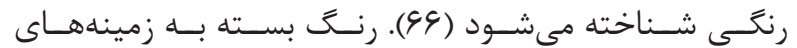

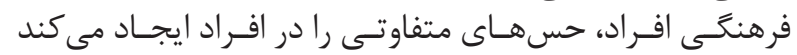

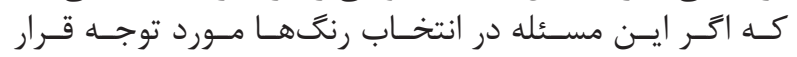

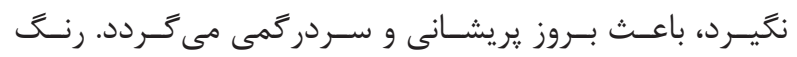

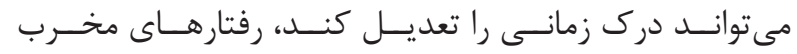

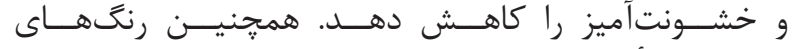

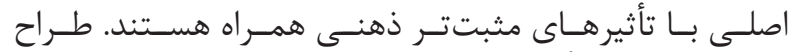

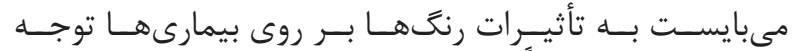

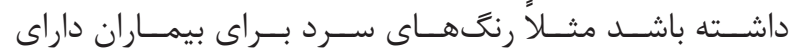

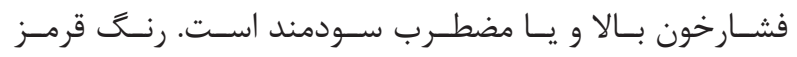

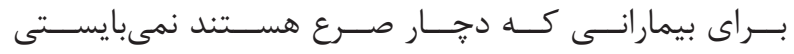

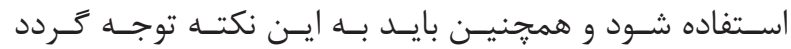

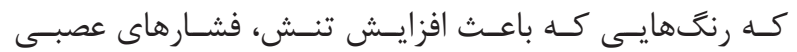

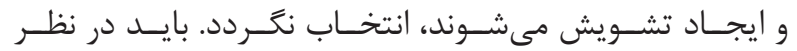

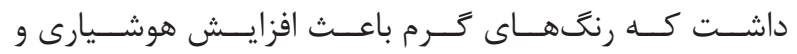

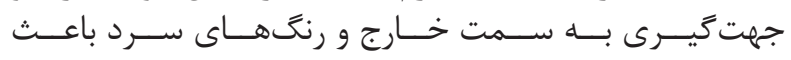

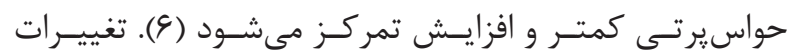

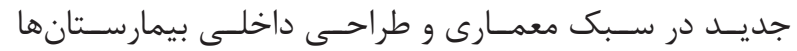

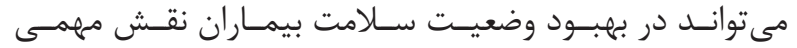

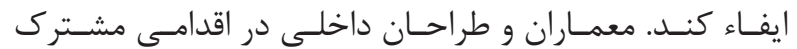

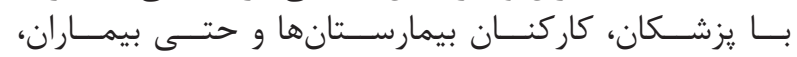

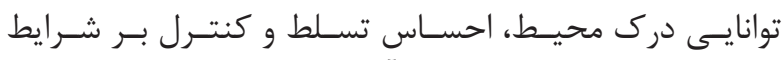

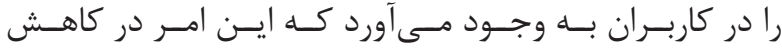

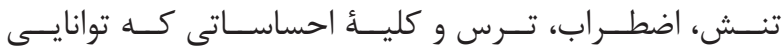

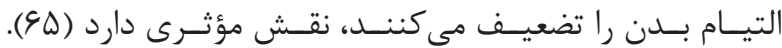

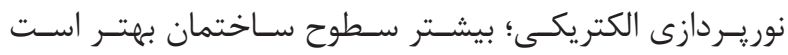

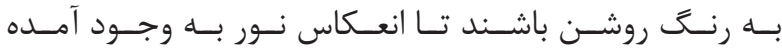

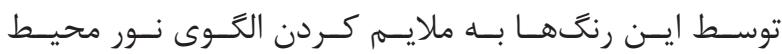

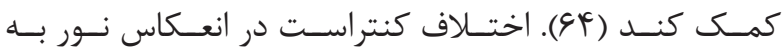

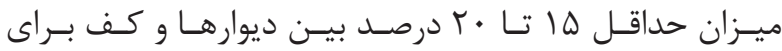

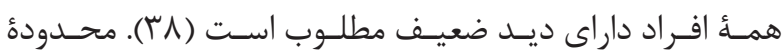

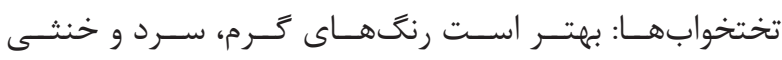

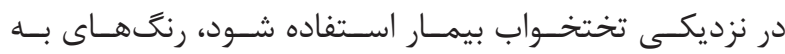

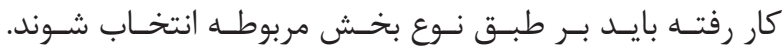

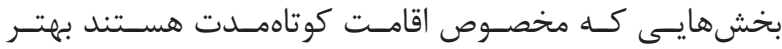

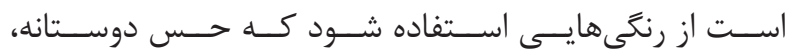

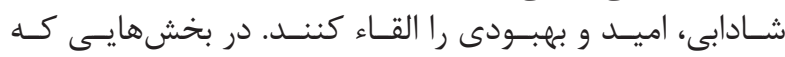

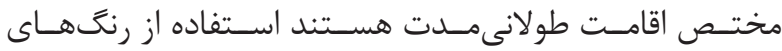

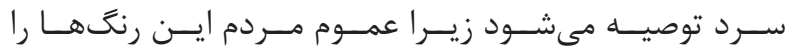

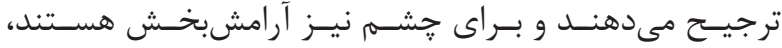

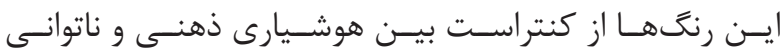

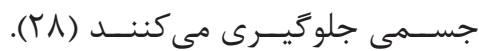

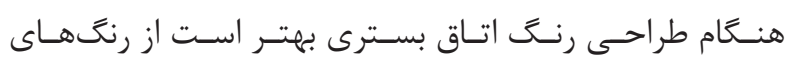

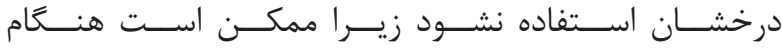

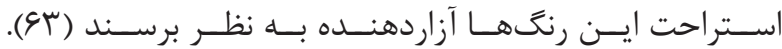

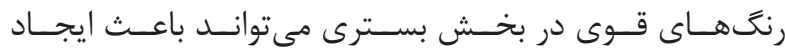

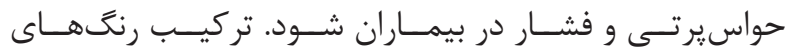

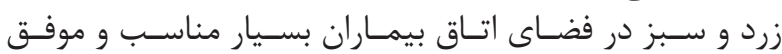

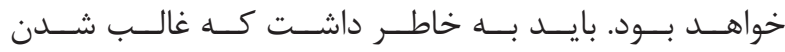

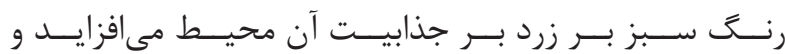

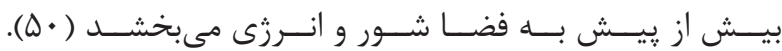

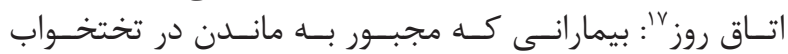

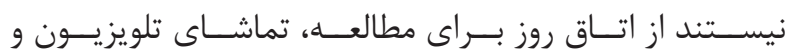

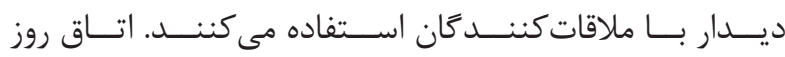

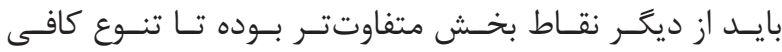

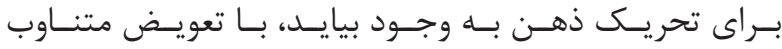

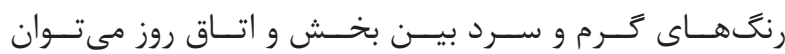

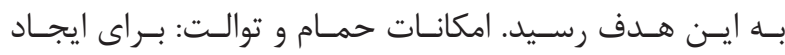

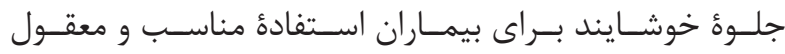

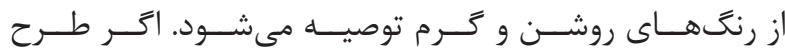

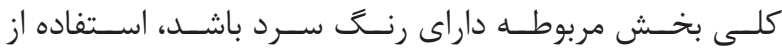

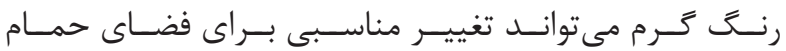

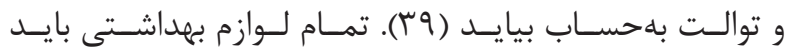

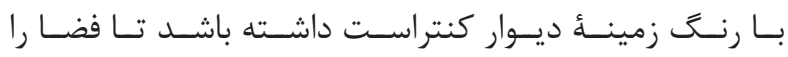

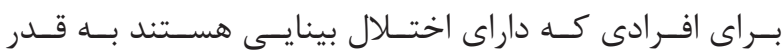

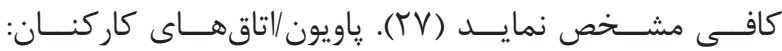

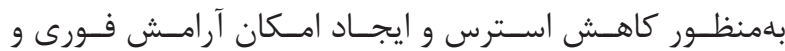

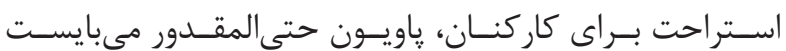




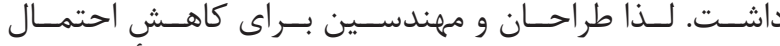

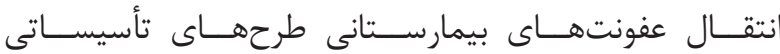

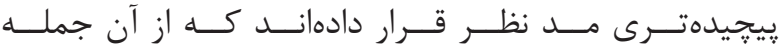

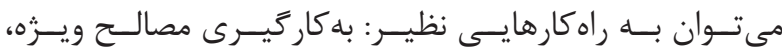

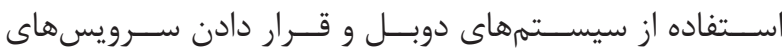

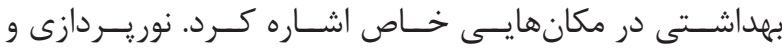

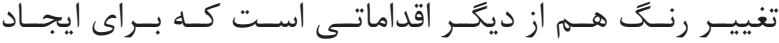

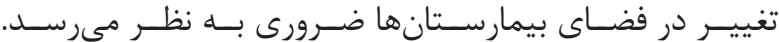

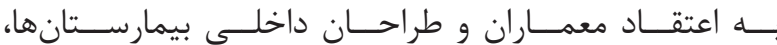

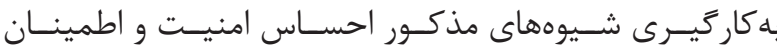

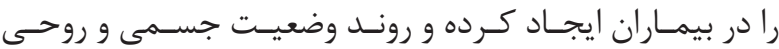

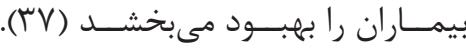

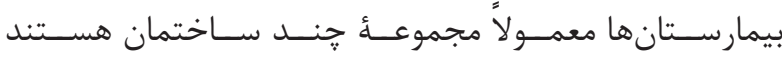

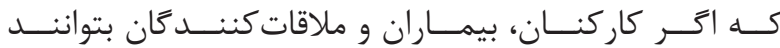

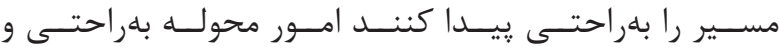

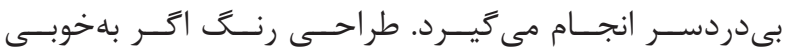

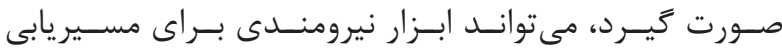

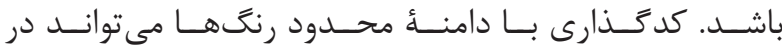

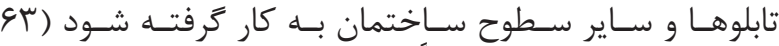

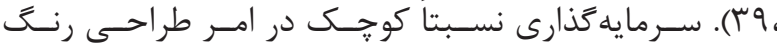

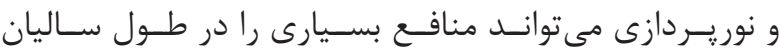

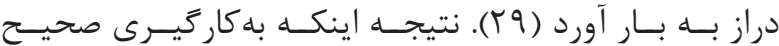

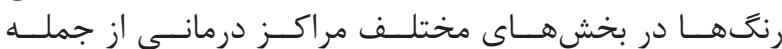

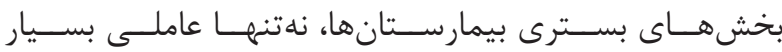

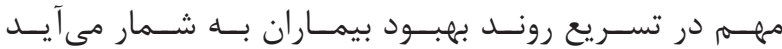

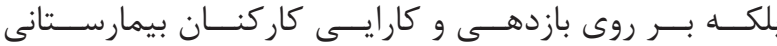

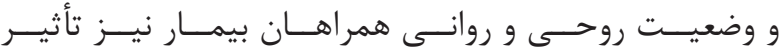
جشــمخيرى دارد.

1. Dilouie C. quality metrics. architectural lighting. 1996; p. 50-3.

\section{Itten J. The art of color. Tehran:Yesavoli. 1999.}

3. Kolivand P, Kazemi H. Lighting and color for hospital design. Mir Mah Publication. 2012.

4. LeGrice M. Experimental cinema in the digital age. London: Britis Film Institute. 2001.

5. Atkinson RL, Atkinson RC. Hilgard's introduction to psychology. International Thomson Publishing. 1999.

6. Gorji Mahlabani Y, Saleh Ahangar M. The effect of color in the design of the hospital. Iranian Architecture and Urbanism. 2014; 1(6): 61-75.

7. Faber B. Color psychology and color therapy. New Hyde Park: University Books, Inc. 1961.

8. Educational film. http://en.wikipedia.org/wiki/
درصـدد يافتـن راه كارهــاى جديـد در تغييـر ســــ معمــارى

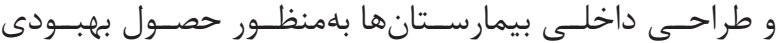

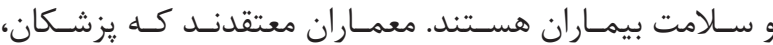

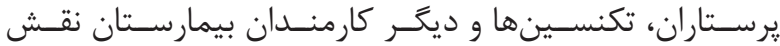

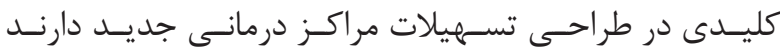

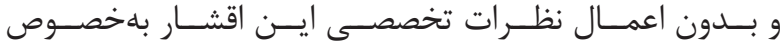

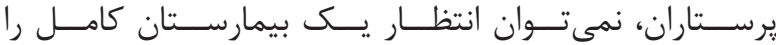

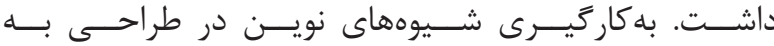

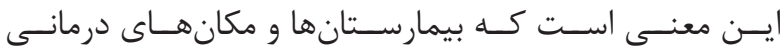

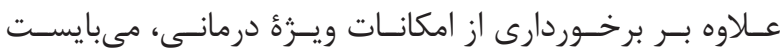

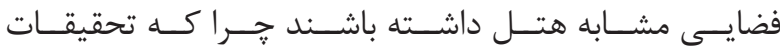

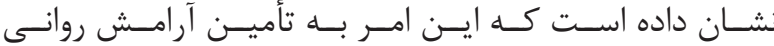

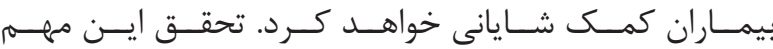

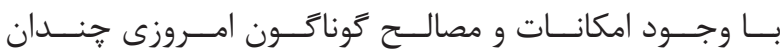

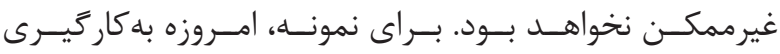

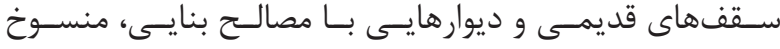

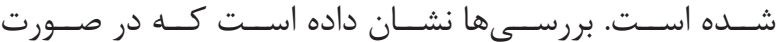

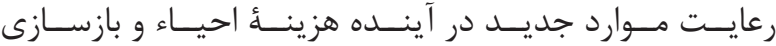

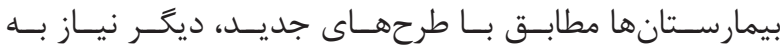

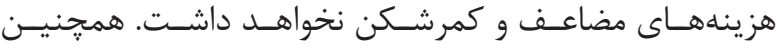

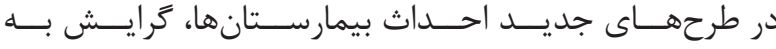

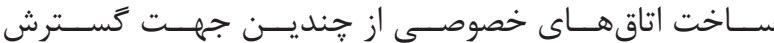

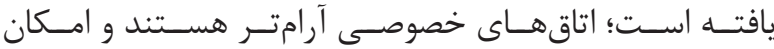

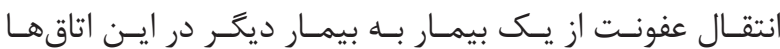

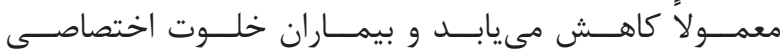

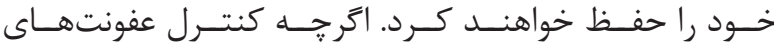

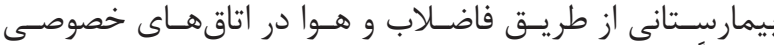

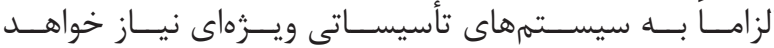

منابع

Educational_film.

9. Edge KJ. Wall color of patient's room: Effects on recovery. Master Thesis. University of Florida. 2003.

10. The effect of colors on human communication. http:// www.migna.ir/vdchtknxd23nq.ft2.html.

11. Khouw N. The meaning of color for gender. http:// www.colormatters.com/khouw.html.

12. Martin CL. The role of cognition in understanding gender effects. Adv Child Dev Behav. 1991; 23: 113-49.

13. Birren F. Color and human response: Aspects of light and color bearing on the reactions of living things and the welfare of human beings. $1^{\text {st }}$ ed. Wiley. 1984.

14. Ford R. How I woke up presentation in with design in mind in RIBA in 19/06/02. London: Isle of Wight Healthcare NHS Trust. 2002.

15. The Facilities Management Team. Personal interview, 
Guy's and St Thomas’s hospital. London. 2002.

16. Conway BR. Color vision, cones, and color-coding in the cortex. Neuroscientist. 2009; 15(3): 274-90.

17. Zhao X, Zhong Y, Zhao Y. Research and analysis on non-medical approaches to alleviate fatigue) international conference on global economy, commerce and service science (GECSS 2014) . Published by Atlantis Press. 2014: p. 253-5.

18. Samina T, Azeemi Y, Mohsin Raza S. A critical analysis of chromotherapy and its scientific evolution. Evid Based Complement Alternat Med. 2005; 2(4): 481-8.

19. Klotsche C. Colour medicine. Arizona: Light Technology Publishing. 1993.

20. Coclivo A. Coloured light therapy: overview of its history, theory, recent developments and clinical applications combined with acupuncture. Am J Acupunct. 1999; 27: 71-83.

21. Demarco A, Clarke N. An interview with Alison Demarco and Nichol Clarke: light and colour therapy explained. Complement Ther Nurs Midwifery. 2001; 95: 103-7.

22. Colour history. Accessed at http://www. colourtherapyhealing.com/colour/colour_history.php

23. Mazuch R. Healing with design. HD: The Journal for Healthcare Design \& Development. 2000; p. 11.

24. Bright $\mathrm{K}$, Cook G. The colour, light and contrast manual: designing and managing inclusive built environments. $1^{\text {st }}$ ed. Wiley-Blackwell. 2010.

25. Baker S. Environmentally friendly? London: Mind. 2004.

26. Estates NHS. The architectural healthcare environment and its effects on patient health outcomes: A report on an NHS estates funded research project. Stationery Office Books. 2003.

27. Bright K, Cook G, Harris J. Colour selection and the visually impaired-a design guide for building refurbishment. http://www.reading.ac.uk/kqFINCH/nhe/ research/rainbow/rainbow.htm.

28. Schuschke G, Christiansen H. Patient-related color preference and color design in the hospital. Zentralbl Hyg Umweltmed. 1994; 195(5-6): 419-31.

29. Guilford JP, Smith PC. A system of color-preferences. Am J Psychol. 1959; 72: 487-502.
30. Mahmoudi K, Shakibamanesh A. Chromatics principles in architecture and urbanism. Heleh publication. 2005.

31. Störring M, Andersen HJ, Granum E. skin color detection under changing lighting conditions. $7^{\text {nd }}$ ed. Symposium on Intelligent Robotics Stems. 1999.

32. Bright K, Cook G, Harris J. Colour and contrast-A design guide for the use of colour and contrast to improve built environments for visually impaired people. Billingham. 1997.

33. Orchard SE. Proceedings of the inter-society color council technical conference "Instrumental Colorant Formulation 1976" Williamsburg, Virginia II. Color Res Appl. 1977; 2(1): 26-31.

34. Tabatabaei MA. Hospital design criteria. http:// tabatabaei66.persianblog.ir/post/15/.

35. Valadi A. Application and concept of color. http:// www.naghsh-negar.ir.

36. Hojati MA. Educational effect of color: how to use color in life. Jamal Publication. 2004.

37. Birren F. Color psychology and color therapy: A factual study of the influence of color on human life. Martino Fine Books. 2013.

38. Wijk H, Berg S, Sivik L, Steen B. Color discrimination, color naming and color preferences in 80-year olds. Aging (Milano). 1999; 11(3): 176-85.

39. Dalke H, Camgoz N, Bright K, Cook G, Loe D. Research with users: colour design and lighting for public transport, Prison and health care environments. 2003.

40. Ardalani H, Ardalani M. Practical tips about the properties of paints in the hospital. http:/hospital-ir.com/ articledetails/3371/.

41. Saito M. Blue and seven phenomena among Japanese students. Percept Mot Skills. 1999. 89(2): 532-6.

42. Duncan MJ. Designed to heal: architecture of Swedish clinic is about more than medicine. Mod Healthc. 2000; 30(47): 32-4.

43. Luscher M, Scott L. The luscher color test. New York: Random House. 1969.

44. Vernolia C. Healing environments. Berkeley: Celestial Arts. 1988.

45. MacDonald LW. Using color effectively in computer graphics. IEEE Comput Graph Appl. 1999; 19(4): 20-35. 


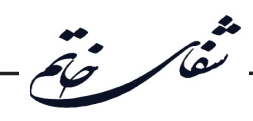

46. Inclusive transport environment: colour design, lighting and visual impairment. http://www.reading. ac.uk/kqFINCH/nhe/research/fit/fit.htm.

47. Pasto T, Kivisto P. Group differences in color choice and rejection. J Clin Psychol. 1956; 12: 397-81.

48. Sadr SS, Arbabi M, Behbood J. Preferences and disgust of color in schizophrenic patients and healthy people. 2004 ; 8(1): 10-6.

49. Pierman BC. Color in the health care environment. Proceedings of a special workshop held at the national bureau of standards, Gaithersburg, Maryland. Washington: Department of Commerce. 1976.

50. Nilgun Camgoz N. Effects of hue, saturation, and brightness on attention and preeference. $\mathrm{PhD}$ thesis. Bilkent University. 2000.

51. Inui M, Miyata T. Spaciousness in interiors. Lighting Research and Technology. 1973; 5(2): 103-11.

52. Estenvi A, Pahlavan P. Shefa Encyclopedia: 32 exotic treatment. Tehran publication. 2004.

53. Bosch SJ, Cama R, Edelstein E, Malkin MJ. The application of color in healthcare settings. www.ki.com/ pdfs/Color_in_Healthcare_Settings_Paper.pdf.Date access: $20 / 3 / 2014$

54. Barker P, Fraser J. Sign design guide: a guide to inclusive signage. London: JMU; Harpenden: Sign Design Society. 2004.

55. Ulrich RS. View through a window may influence recovery from surgery. Science. 1984; 224(4647): 420-1.

56. Shamgholi SH. Basic concepts in architectural design of hospitals. Soroush Danesh Press. 2011.

57. Dotton FJ, Mandleco B. Child and family communication. In pediatric nursing. Caring for children and their families. 3rd ed. Delmar, Clifton Park, NJ: Delmar. 2012; p. 409-31

58. Lowenhaupt CB. Windows and people: a literature survey: psychological reaction to environments with and without windows. National Bureau of Standards (US) publisher. 1975.

59. Minnaert M. Light and color in the outdoors. New York, Springer. 1995; p. 293.

60. Inui M, Miyata T. Spaciousness behavior and the visual environment. J Light Vis Environ. 1977; 1: 59-63.

61. Leather P, Beale D, Santos A, Watts J, Lee L. Outcomes of environmental appraisal of different hospital waiting areas. Environ Behav. 2003; 35(6): 842-69.

62. National Health Service Estates. Way finding: effective way finding and signing systems: Guidance for healthcare facilities. TSO. 1999.

63. Gill JM. Which button? Designing user interfaces for people with visual impairments. Royal National Institute for the Blind. 2000; p. 28.

64. Hospital Design. http://hospital-ir.com/news/96.

65. Arthur P, Passini R. Wayfinding: People, signs and architecture. New York: McGraw-Hill Inc. 1992.

66. Daggett WR, Cobble JE, Gerfel SJ. Color in an optimum learning environment. International Center for leadership in Education. 2008; p. 1-9. 